\title{
Der Politikverflechtungsfalle entwischt? Die Effekte der Föderalismusreform I auf die Gesetzgebung*
}

\author{
Kurzfassung
}

Wie hat sich die Föderalismusreform I auf die Gesetzgebung in Deutschland ausgewirkt? Für die Bundesländer zeigt sich, dass die neu gewonnenen Kompetenzen begrenzt sind und die Länder in erheblichem Maße zu einer horizontalen Koordinierung greifen. Auf Bundesebene ging der Anteil zustimmungsbedürftiger Gesetze in den ersten 28 Monaten nach Inkrafttreten der Reform in nennenswertem Umfang zurück, wobei allerdings vornehmlich Routineentscheidungen vom Wegfall der Zustimmungsbedürftigkeit betroffen zu sein scheinen. Eine kontrafakische Analyse der Schlüsselentscheidungen der rot-grünen Koalition bestätigt den Befund einer abnehmenden Zustimmungsquote, zeigt aber zudem, dass auch einige Richtungsentscheidungen vermutlich zukünftig nicht mehr die Zustimmung des Bundesrates benötigen. Dennoch ist nicht mit einer massiven Veränderung der Willensbildungsprozesse zu rechnen, da der Parteienwettbewerb weiterhin kohärente Reformpolitik zu verhindern droht. Zudem hängen die Effekte der Reform in erheblichem Umfang von der Interpretation der einschlägigen Grundgesetzartikel durch das Bundesverfassungsgericht $a b$.

* Der Beitrag ist die schriftliche und überarbeitete Fassung meines Habilitationsvortrages vor der Fakultät für Wirtschafts- und Sozialwissenschaften der Ruprecht-KarlsUniversität Heidelberg. Für hilfreiche Anmerkungen danke ich Klaus von Beyme, Christoph Egle, Lars P. Feld, Sebastian Harnisch, Marcus Höreth, Sven Leunig, Carolyn Moore, Manfred G. Schmidt, Uwe Wagschal, Frieder Wolf sowie den zwei anonymen Gutachtern. 


\section{Inhalt}

1. Einleitung

2. Föderalismusreform und Gesetzgebung: Ein Überblick über die wichtigsten Veränderungen

3. Ein Blick auf die Länder

4. Die Zustimmungsbedürftigkeit von Bundesgesetzen seit Inkrafttreten der Föderalismusreform

5. Die Schlüsselentscheidungen der rot-grünen Bundesregierung - eine kontrafaktische Analyse

6. Stimmen die Annahmen? Föderalismusreform und der politische Prozess

7. Fazit: Der Politikverflechtungsfalle entwischt?

\section{Einleitung}

Am 01. September 2006 ist die sogenannte Föderalismusreform I in Kraft getreten. Dabei handelt es sich um die bislang umfangreichste Änderung des Grundgesetzes (Busch 2006: 42; Reutter 2006: 1250). 25 Grundgesetzartikel wurden geändert, aufgehoben oder neu eingeführt. Die Erwartungen an diese Reform, nicht zuletzt seitens der beteiligten Entscheidungsträger, waren hoch. Einer der beiden Vorsitzenden der Kommission, die die erste Stufe der Föderalismusreform vorbereiten sollte, der seinerzeitige bayerische Ministerpräsident Edmund Stoiber, sprach gar von der „Mutter aller Reformen“ (zitiert bei Scharpf 2007b: 200). Von verschiedenen Seiten wurde die Hoffnung gehegt, dass mit einer Reform des Föderalismus die leidigen Probleme der Politikverflechtung gelöst und der viel beschworene Reformstau in der deutschen Politik aufgelöst werden könnten. Die Politikverflechtung, also die Notwendigkeit für Bund und Länder, die meisten wichtigen politischen Entscheidungen gemeinsam zu treffen, sei nämlich verantwortlich dafür, dass das deutsche politische System systematisch suboptimale Entscheidungen treffe, die sich in zu langsamer und zu wenig weit reichender Anpassung an veränderte wirtschaftliche, gesellschaftliche und kulturelle Rahmenbedingungen niederschlügen. Die Politikverflechtung - so die Analyse - führe zu „Reformstau“. Entweder käme es erst gar nicht zu Entscheidungen oder diese 
seien durch die Vielzahl der an ihnen beteiligten Akteure so weit verwässert, dass sie ihren Zweck kaum erfüllen könnten.

Für viele Beobachter wie auch Entscheidungsträger war demnach der Lösungsweg zur Beendigung des Reformstaus klar: Eine Entflechtung musste her. Im Bereich der Gesetzgebung, um die es im Folgenden gehen soll, hieß das: Einerseits sollten die Gesetzgebungskompetenzen der Länder erweitert werden, damit auf dieser Ebene in größerem Umfang eigenständig gehandelt werden kann; andererseits sollten im Gegenzug die Länder, genauer gesagt: die im Bundesrat vertretenen Länderregierungen, dann aber wenigstens in Teilen auf ihre Vetorechte in Bezug auf die Bundesgesetzgebung verzichten. Im Gegenzug für eine Ausweitung der Gesetzgebungskompetenzen der Länder sollte also der Anteil der im Bundesrat zustimmungsbedürftigen Gesetze verringert werden.

Die Frage ist nun, ob dies mit der Föderalismusreform I gelungen ist, ob also tatsächlich eine legislative Entflechtung erreicht worden ist. Genau dieser Frage nach den Auswirkungen der Föderalismusreform auf die Gesetzgebung, hauptsächlich auf die Gesetzgebung der Bundesebene, soll der Beitrag empirisch nachgehen.

$\mathrm{Zu}$ Beginn wird daher knapp auf die wichtigsten für die Gesetzgebung relevanten Neuerung der Föderalismusreform eingegangen (2.). Danach wird ein kurzer Blick auf die Länderebene geworfen, um zu überprüfen, ob dort tatsächlich ein größerer Gestaltungsspielraum geschaffen wurde und wie er genutzt wird (3.). Anschließend - und das bildet den Hauptteil des Beitrags werde ich mich mit den Auswirkungen der Föderalismusreform auf die Gesetzgebung auf Bundesebene beschäftigen. Das geschieht auf dreierlei Arten. Ich werde zunächst schlicht die Gesetzgebung der ersten 28 Monate seit Inkrafttreten der Föderalismusreform daraufhin untersuchen, wie sich der Anteil der zustimmungsbedürftigen Gesetze an allen verabschiedeten Gesetzen verändert hat und welche Gesetze gegebenenfalls der Zustimmung des Bundesrates nicht mehr bedurften (4.). In einem zweiten Schritt werde ich mittels einer kontrafaktischen Analyse untersuchen, wie sich die Föderalismusreform auf die Politik der rot-grünen Bundesregierung ausgewirkt hätte, wenn die Reform bereits 1998 in Kraft gewesen wäre. Ich werde also überprüfen, inwieweit sich die Zustimmungsbedürftigkeit von rund 70 Schlüsselentscheidungen der rot-grünen Bundesregierung verändert hätte, wenn für diese Entscheidungen bereits die durch die Föderalismusreform neu bestimmten Regeln gegolten hätten (5.). In einem dritten Schritt schließlich frage ich dann, ob die zentrale Grundannahme, auf der die Föderalismusreform I 
basiert, zutrifft, dass die Politikverflechtung für den vermeintlichen Reformstau in der deutschen Gesetzgebung verantwortlich ist. Wiederum am Beispiel der Politik unter der rot-grünen Bundesregierung werde ich zu zeigen versuchen, dass dies keineswegs zutrifft, und dass es zumindest noch weitere Gründe für die häufig langwierige und manchmal träge Reformtätigkeit in der deutschen Politik gibt, denen die Föderalismusreform jedoch nicht zuleibe gerückt ist, und denen sie auch gar nicht zuleibe rücken konnte (6.). Im abschließenden Fazit wird die Frage diskutiert, ob die vorgestellten Ergebnisse es erlauben, davon zu sprechen, mit der Reform hätten die Akteure der Politikverflechtungsfalle ein Schnippchen geschlagen (7.).

\section{Föderalismusreform und Gesetzgebung: Ein Überblick über die wichtigsten Veränderungen}

Die wichtigsten Veränderungen, die sich bei der Gesetzgebung durch die Föderalismusreform I ergeben haben, können in sechs Punkten zusammengefasst werden.

1. Die Rahmengesetzgebung wurde vollständig abgeschafft. Die meisten betroffenen Bereiche - eine wichtige Ausnahme bilden die meisten Materien des Hochschulrechts mit Ausnahme der Hochschulzulassung und der Hochschulabschlüsse - gingen in die konkurrierende oder die ausschließliche Gesetzgebungskompetenz des Bundes über. ${ }^{1}$

2. Sechzehn, häufig allerdings eng umgrenzte Regelungsmaterien wurden in die ausschließliche Gesetzgebungskompetenz der Länder übertragen.

3. Bei der konkurrierenden Gesetzgebung kam es zu erheblichen Änderungen. So gilt die Erforderlichkeitsklausel des Art. 72 Abs. 2 GG, nach der dem Bund das Gesetzgebungsrecht in der konkurrierenden Gesetzgebung nur zusteht, wenn und soweit die Herstellung gleichwertiger Lebensverhältnisse oder die Wahrung der Rechts- oder Wirtschaftseinheit eine bundesgesetzliche Regelung erforderlich macht, nur noch für zehn der 32 Bereiche der konkurrierenden Gesetzgebung. In den anderen 22 Bereichen kann der Bund Gesetze erlassen, ohne die Erforderlichkeit nachzuweisen. Allerdings haben die Länder im Gegenzug bei sechs dieser 22 Materien, die allesamt vor der Reform unter die Rahmengesetzgebungskompetenz des Bundes gefallen waren, absolute Abweichungsrechte, d. h. die Länder können in diesen Bereichen

1 Unter anderem wurde auf diese Weise die Schaffung eines Umweltgesetzbuches ermöglicht. 
trotz des Bestehens einer bundesgesetzlichen Regelung Gesetze erlassen, die dann auch dem Bundesrecht vorgehen.

4. Dem Bund wurden einige wenige neue Gesetzgebungskompetenzen übertragen. Am wichtigsten ist dabei die neue ausschließliche Gesetzgebungskompetenz zur Abwehr des internationalen Terrorismus durch das Bundeskriminalpolizeiamt in drei Fällen (Art. 73 Abs. 1 Nr. 9a GG). ${ }^{2}$

5. Verloren hat der Bund die ausschließliche Gesetzgebungskompetenz über die Gemeinschaftsaufgabe Ausbau und Neubau von Hochschulen einschließlich der Hochschulkliniken (Art. 91a GG), die ersatzlos gestrichen wurde.

6. Durch eine Änderung von Art. 84 Abs. 1 GG sollte die Zahl der im Bundesrat zustimmungsbedürftigen Gesetze gesenkt werden. Gleichzeitig wurde jedoch mit dem neuen Art. 104a Abs. 4 GG eine zusätzliche, die Zustimmungsbedürftigkeit auslösende Norm geschaffen. Auf diese beiden Veränderungen wird später noch ausführlicher einzugehen sein.

Bevor ich mich aber eingehender mit der Frage beschäftige, wie sich die Föderalismusreform I auf die Gesetzgebung auf Bundesebene, und insbesondere auf die Zustimmungsbedürftigkeit von Bundesgesetzen ausgewirkt hat, will ich einen kurzen Blick auf die Länderebene werfen.

\section{Ein Blick auf die Länder}

Wirft man einen Blick auf die Veränderungen, die sich durch die Reform für die Gesetzgebung der Länder ergeben haben, zeigt sich, dass der auf dieser Ebene gewonnene Handlungsspielraum vermutlich kleiner ist, als es die schiere Zahl von 16 Regelungsmaterien erscheinen lässt, die neu in die ausschließliche Gesetzgebungskompetenz der Länder übertragen worden sind. Zwar ist die neu auf die Länder übertragene Gesetzgebungskompetenz für die Besoldung und Versorgung der Angehörigen des öffentlichen Dienstes von einiger Bedeutung - und auch politischer Brisanz. Diese Regelung war es auch, deretwegen die Länder Mecklenburg-Vorpommern (Ablehnung) und Schleswig-Holstein (Enthaltung) der Föderalismusreform im Bundesrat die Zustimmung vorenthielten (Bundesrat, 824. Sitzung, 07.07.06, S. 216, 217, 221, 222). Die meisten übrigen neu errungenen Länderkompetenzen sind dagegen von eher begrenztem politischen Gewicht, wenn man etwa an

2 Auf der Basis dieser neuen Gesetzgebungskompetenz konnte das „Gesetz zur Abwehr von Gefahren des internationalen Terrorismus durch das Bundeskriminalamt", das sogenannte BKA-Gesetz, verabschiedet werden (vgl. BT-Drs. 16/10121: 16). 
das Heimrecht, das Recht der Flurbereinigung oder den Ladenschluss und das Gaststättenrecht denkt. Auch zur neuen Länderkompetenz beim Versammlungsrecht, die ja zunächst durchaus gewichtig erscheint, heißt es in einem juristischen Kommentar zur Föderalismusreform: „Allerdings ist der Gestaltungsspielraum der Länder [...] durch die äußerst intensive grundrechtliche Durchdringung der Materie durch die Rechtsprechung des Bundesverfassungsgerichts und des Bundesverwaltungsgerichts zu Art. 8 GG erheblich beschränkt" (Kluth 2007: 176). In ähnlicher Weise ist auch die Reichweite der Abweichungsrechte bei Hochschulzulassung und Hochschulabschlüssen begrenzt, da hier nennenswerte Festlegungen existieren, und zwar durch das Numerus-Clausus-Urteil des Bundesverfassungsgerichts im Bereich der Hochschulzulassung und durch die von der Kultusministerkonferenz vereinbarten ländergemeinsamen Strukturvorgaben aus dem Jahr 2003 im Bereich der Hochschulabschlüsse (Münch 2008: 235).

In der Öffentlichkeit spielte allerdings ohnehin die Regelung, dass den Ländern nun mit Ausnahme der Hochschulzulassung und der Hochschulabschlüsse die Gesetzgebungskompetenz für die gesamte Bildungspolitik übertragen wurde, die prominenteste Rolle. Allerdings dürften auch diese Änderungen von geringerer Bedeutung sein, als von vielen erwartet, wie insbesondere an der Schulpolitik zu erkennen ist: „Schon vor der Föderalismusreform 2006 war der schulpolitische Einfluss des Bundes gering" (Rürup 2008: 174; ähnlich Wolf 2008: 21). Allenfalls die Steuerung der Landesschulpolitiken durch finanzielle Anreize des Bundes dürfte durch den neuen Artikel 104b GG erschwert worden sein, der nämlich Finanzhilfen des Bundes nur noch dann erlaubt, wenn dieser in dem entsprechenden Bereich auch Gesetzgebungskompetenzen besitzt. Allerdings zeigte bereits die Finanzierung des Kinderbetreuungsausbaus unter der Großen Koalition, dass auch das vermeintlich strikte Verbot von Finanzhilfen im Bildungsbereich mit Hinweis auf die wachstumsfördernden Effekte von Bildung und die Gesetzgebungskompetenz des Bundes für die öffentliche Fürsorge unschwer umgangen werden kann. ${ }^{3}$

3 Konkret errichtete der Bund ein Sondervermögen „Kinderbetreuungsausbau“. Die Gesetzesbegründung führt aus: „Rechtsgrundlage hierfür wird Artikel 104b des Grundgesetzes (GG) sein. Mit den Finanzmitteln sollen besonders bedeutsame Investitionen gefördert werden, die erforderlich sind, die strukturellen Bedingungen für die Wirtschaftsentwicklung in Deutschland zu verbessern und damit das wirtschaftliche Wachstum zu fördern. Das Grundgesetz verleiht dem Bund hierfür die Gesetzgebungsbefugnis nach Artikel 74 Abs. 1 Nr. 7 GG (öffentliche Fürsorge)“ (BT-Drs. 16/6596: 3). Ob diese Argumentation verfassungskonform ist, sei allerdings dahingestellt. 
Hinzu kommt, dass die Länder wenigstens in den ersten zwei Jahren nach Inkrafttreten der Föderalismusreform ihre neu errungenen Gesetzgebungsbefugnisse offenbar nur in begrenztem Umfang verwendet haben (zum Folgenden vgl. vor allem BT-Drs. 16/8688 vom 02.04.08, S. 9-11 sowie Leunig 2008). In Bezug auf ihr absolutes Abweichungsrecht bei bestimmten Materien der konkurrierenden Gesetzgebung liegt das daran, dass die Länder aufgrund von Übergangsfristen in einigen wichtigen Bereichen etwa des Umweltrechts bis zum 01.01.10 sowie bei Hochschulzulassung und Hochschulabschlüssen bis zum 01.08.08 noch gar nicht von Bundesrecht abweichen dürfen bzw. durften (Art. 125b Abs. $1 \mathrm{GG}$ ). Aber auch dort, wo die Länder nicht auf diese Weise eingeschränkt waren, ist die Gesetzgebung nur schwer in Schwung gekommen. Lediglich beim Jugendstrafvollzug, beim Nichtraucherschutz in Gaststätten und beim Ladenschluss haben alle Länder, im Fall des Ladenschlussgesetzes alle Länder außer Bayern, ein eigenes Gesetz beschlossen. Dabei ist allerdings für den Fall der Jugendstrafvollzugsgesetze einschränkend darauf hinzuweisen, dass das Bundesverfassungsgericht dem Gesetzgeber, und damit seit Inkrafttreten der Föderalismusreform den Landtagen, für den Erlass eines Jugendstrafvollzugsgesetzes eine Frist bis Ende 2007 gesetzt hatte (BVerfGE 116: 69 ff.), was erklärt, weshalb hier alle Länder aktiv werden mussten.

In allen anderen Politikfeldern haben die Länder eher zögerlich auf ihre neuen Gesetzgebungskompetenzen reagiert. Das gilt sogar für die Hochschulpolitik, für die Sandberger (2008: 171) festhält: „Die gewonnene Gestaltungsfreiheit wird nur zögernd aufgenommen und nur in Teilbereichen, wie der Gestaltung der Personalstruktur oder des Zulassungswesens, realisiert.“ Lediglich Baden-Württemberg hat bereits ein Gesetz zur Umsetzung der Föderalismusreform im Hochschulbereich verabschiedet. Der aktuelle Reformpfad in der Hochschulpolitik der anderen Länder (und auch in BadenWürttemberg) geht dagegen bereits auf die Zeit vor der Föderalismusreform zurück und wäre daher in den meisten Bereichen auch ohne diese möglich gewesen (Lanzendorf/Pasternack 2008: 51 ff.).

Beim Laufbahnrecht wollten die Länder dagegen auf die Verabschiedung des Beamtenstatusgesetzes des Bundes warten (BT-Drs. 16/8688: 9), das erst Ende April 2008 verabschiedet worden ist, aber auch im Bereich der Besoldung und Versorgung haben die Länder das geltende Recht zumindest nicht vollständig ersetzt, sondern in der Regel nur Besoldungsanpassungsgesetze verabschiedet. In anderen Bereichen haben allenfalls einzelne Länder Gesetze erlassen, so beispielsweise Baden-Württemberg, Bayern und 
Nordrhein-Westfalen im Heimrecht, Baden-Württemberg, Bayern und Hamburg im Wohnungswesen (Rahm 2008). In Bayern, Hamburg und Niedersachsen sind darüber hinaus neue Strafvollzugsgesetze verabschiedet worden (Kinzig/Steinhilber 2008). Ein vollständiges Versammlungsgesetz hat lediglich Bayern bereits verabschiedet, Brandenburg ist in Bezug auf Versammlungen auf Gräberstätten aktiv geworden. Von der Kompetenz, den Steuersatz der Grunderwerbsteuer zu variieren, hat bislang nur Berlin Gebrauch gemacht. In den Bereichen Pressewesen und Lärmbekämpfung schließlich ist noch gar keine Gesetzgebungsaktivität der Länder zu verzeichnen.

Auffallend ist zudem, dass die Länder in einer ganzen Reihe von Fällen eine horizontale Koordination ihrer Politiken vorgenommen haben. Das gilt für den Ladenschluss, bei dem es zumindest zu Verhandlungen über eine bundesweit einheitliche Regelung kam, wenngleich sich die einzelnen Regelungen in den 15 Ländern, die ein eigenes Ladenschlussgesetz verabschiedet haben, nun doch teilweise unterscheiden. Doch auch in anderen Bereichen gab es Bedenken gegen bundesweit differierende Regelungen, vielleicht etwas überraschender Weise nicht zuletzt in Bayern: So sollten beispielsweise nach Ansicht eines Mitglieds der bayerischen Staatsregierung hinsichtlich des Rauchverbots in Gaststätten im baden-württembergischen Ulm keine anderen Regeln gelten als im bayerischen Neu-Ulm (Scharpf 2007a: 18). Entsprechend setzten die Länder bezüglich des Nichtraucherschutzes in Gaststätten eine Arbeitsgruppe ein, die einheitliche Standards erarbeiten sollte, die dann von der Ministerpräsidentenkonferenz im März 2007 wenigstens im Prinzip angenommen wurden (BT-Drs. 16/8688: 10). Ebenso fanden sich neun Länder ${ }^{4}$ zusammen, um einen gemeinsamen Entwurf für ihre Jugendstrafvollzugsgesetze zu erarbeiten - mit dem expliziten Ziel, „möglichst einheitliche Standards für den Jugendstrafvollzug zu sichern - trotz des Föderalismus", so der Justizminister von Schleswig-Holstein, Uwe Döring (SPD), bei der ersten Lesung des entsprechenden Landesgesetzes (PIPr. LT SchleswigHolstein, 16. WP, 64. Sitzung, 12.07.07, S. 4602). Schließlich koordinierten sich sechs Bundesländer ${ }^{5}$ auch bei der Erarbeitung der Landesheimgesetze (Rothgang/Wessel 2008: 168).

4 Berlin, Brandenburg, Bremen, Mecklenburg-Vorpommern, Rheinland-Pfalz, Saarland, Sachsen-Anhalt, Schleswig-Holstein und Thüringen. Sachsen, das dieser Gruppe ursprünglich auch angehört hatte, war im Laufe der Beratungen ausgeschieden.

5 Brandenburg, Bremen, Mecklenburg-Vorpommern, Rheinland-Pfalz, Sachsen-Anhalt und Schleswig-Holstein. 
Fasst man diese Befunde zusammen, wird man sich schwer tun zu schlussfolgern, dass die Übertragung einiger Gesetzgebungskompetenzen auf die Länder eine weitreichende Entflechtung der deutschen Gesetzgebung bewirkt hat. Dazu sind einerseits die übertragenen Kompetenzen sowie die durchgesetzten Änderungen trotz gelegentlicher Pfadabweichungen zu marginal; andererseits wurde die vertikale Politikverflechtung zwischen Bund und Ländern in vielen Fällen aber auch einfach nur durch die horizontale Politikverflechtung zwischen den Ländern, insbesondere den finanzschwachen Ländern ersetzt. Allenfalls die stärkeren Länder haben bereits zum jetzigen Zeitpunkt in nennenswertem Umfang von ihren neuen Gesetzgebungskompetenzen Gebrauch gemacht.

Es bleibt abzuwarten, ob auch die ärmeren Länder im Zeitverlauf beginnen, die neuen Kompetenzen in größerem Umfang als bisher zu nutzen. Das erscheint zumindest denkbar. Wenigstens zum gegenwärtigen Zeitpunkt ist aber davon auszugehen, dass es zu einer Rückführung der legislativen Politikverflechtung auf der Bundesebene hätte kommen müssen, wenn die Reform denn überhaupt $\mathrm{zu}$ einer Entflechtung der Gesetzgebung beitragen sollte. D. h. die Reform hätte zu einer Senkung der Anzahl der Gesetze beitragen müssen, die der Zustimmung des Bundesrates bedürfen.

\section{Die Zustimmungsbedürftigkeit von Bundesgesetzen seit Inkrafttreten der Föderalismusreform}

Der wichtigste Weg, auf dem das Ziel einer Verringerung der Zahl zustimmungsbedürftiger Gesetze erreicht werden sollte, war der neue Artikel 84 Absatz 1 GG. Die alte Fassung dieses Absatzes sah vor, dass Bundesgesetze, die von den Ländern als eigene Angelegenheit ausgeführt werden, dann zustimmungsbedürftig sind, wenn in ihnen die Einrichtung von Behörden oder das Verwaltungsverfahren geregelt wird. Es war dieser Absatz, aus dem sich die Zustimmungsbedürftigkeit von Gesetzen am häufigsten herleitete und auf den sich daher die Arbeit der Föderalismuskommission konzentrierte, die die Reform vorbereitete. Nach der neuen Fassung dieses Absatzes sind nun drei Optionen zu unterscheiden: Erstens kann der Bund auf die Regelung des Verwaltungsverfahrens und der Behördeneinrichtung verzichten. Dann bleibt das Gesetz zustimmungsfrei. Das ist allerdings keine neue Option, diese Möglichkeit bestand auch vor der Föderalismusreform bereits. Die Tatsache, dass Bundesregierungen diesen Weg zur Umgehung des Bundesrates nicht häufiger gewählt haben, spricht allerdings dafür, dass es nicht immer ohne 
Weiteres möglich ist, die materiellen von den verfahrensrechtlichen Regelungen eines Gesetzes zu trennen (vgl. z. B. Scharpf 2006: 2).

Anders als nach alter Rechtslage ist es nun allerdings möglich, die $\mathrm{Zu}-$ stimmung des Bundesrates zu umgehen, auch wenn in dem entsprechenden Gesetz das Verwaltungsverfahren geregelt wird. Dazu muss der Bund dann allerdings akzeptieren, dass die Länder von den im Gesetz vorgesehenen Verwaltungsverfahren abweichen können. Wenn die Bundesregierung jedoch - und das ist die dritte Möglichkeit - auf bundeseinheitliche Verwaltungsverfahren in allen Ländern besteht, ist die Zustimmung des Bundesrates weiterhin erforderlich. Letztere Option, die faktisch weitgehend der alten Rechtslage entspricht, ${ }^{6}$ soll allerdings nach dem Wortlaut nur „,in Ausnahmefällen" gelten.

Während mit dem neuen Art. 84 Abs. 1 also ein Weg gesucht wurde, die Zustimmungsbedürftigkeit von Bundesgesetzen zu reduzieren, hat die Föderalismusreform aber auch einen neuen Zustimmungstatbestand eingeführt. Nach dem neuen Artikel 104a Absatz 4 GG sollen Bundesgesetze zustimmungsbedürftig sein, wenn sie „Pflichten der Länder zur Erbringung von Geldleistungen, geldwerten Sachleistungen oder vergleichbaren Dienstleistungen gegenüber Dritten begründen“ und ,wenn daraus entstehende Ausgaben von den Ländern zu tragen sind". Nach alter Rechtslage trat die Zustimmungsbedürftigkeit erst ein, wenn die Länder mindestens ein Viertel der Kosten zu tragen hatten, und die Regelung bezog sich lediglich auf Geldleistungen, während geldwerte Sachleistungen und die sogenannten ,vergleichbaren Dienstleistungen“ keine Zustimmungsbedürftigkeit auslösten.

Wie haben sich die fraglichen Regelungen in den ersten 28 Monaten seit Inkrafttreten der Föderalismusreform aber tatsächlich ausgewirkt? Ist die Quote zustimmungsbedürftiger Gesetze an allen Gesetzen tatsächlich halbiert worden, wie eine Studie des Wissenschaftlichen Dienstes des Bundestages aus dem Jahr 2006 prognostizierte (Georgii/Borhanian 2006)? Oder hatten die Kritiker recht, die annahmen, dass es wegen der Neufassung des Artikels 104a Abs. 4 GG sogar zu einer weiteren Steigerung der Anzahl zustimmungsbedürftiger Gesetze kommen würde (Scharpf 2007b: 201; Höreth 2007: 732)?

Um dies herauszufinden, habe ich alle seit dem 1. September 2006 im Bundesgesetzblatt verkündeten Gesetze gesammelt und auf ihre Zustimmungs-

6 Eine bundesgesetzliche Regelung der Einrichtung der Behörden ohne Abweichungsmöglichkeit ist nach der neuen Rechtslage allerdings überhaupt nicht mehr möglich. 
bedürftigkeit hin untersucht. ${ }^{7}$ Demnach sind zwischen dem 1. September 2006 und dem 31. Dezember 2008354 Gesetze im Bundesgesetzblatt verkündet worden. Von diesen 354 Gesetzen benötigten 139 die Zustimmung des Bundesrates, während die restlichen 215 ohne die Zustimmung des Bundesrates verabschiedet werden konnten. Das entspricht einer Zustimmungsquote von 39,3\%. Meine Auswertung über die ersten 28 Monate seit Inkrafttreten der Reform unterstützt demnach der Tendenz nach frühere Untersuchungen über kürzere Zeiträume: So ergab eine Aufstellung der Bundesregierung (BT-Drs. 16/8688), dass der Anteil zustimmungsbedürftiger Gesetze an allen im ersten Jahr nach Inkrafttreten der Föderalismusreform I verkündeten Gesetzen bei 44,2 \% gelegen hat. In die gleiche Richtung weisen auch die Ergebnisse von Horst Risse (2007: 709), der allerdings eine etwas andere Datenbasis als die Bundesregierung und ich betrachtet, nämlich den Anteil zustimmungsbedürftiger Gesetze an allen Gesetzesvorlagen, mit denen sich der Bundesrat zwischen September 2006 und September 2007 beschäftigte - diese müssen nicht notwendigerweise verabschiedet worden sein, sodass sie dann auch nicht im Bundesgesetzblatt auftauchen. Risse findet, dass $42,7 \%$ dieser Vorlagen die Zustimmung der zweiten Kammer benötigten. Schließlich ergibt sich bei der Durchsicht der Dokumentation des Bundesrates zu den „Gemäß den Regelungen der Föderalismusreform I behandelten verkündeten Gesetzen“ (Stand 08.12.08) ${ }^{8}$ ein Anteil zustimmungsbedürftiger Gesetze von $38,7 \%{ }^{9}$

7 Um die Vollständigkeit und die Richtigkeit der Zuordnung, ob ein Gesetz zustimmungsbedürftig oder zustimmungsfrei gewesen ist, sicherzustellen, wurden die Ergebnisse mit der Datenbank des Deutschen Bundestages und den Daten, die die Bundesregierung für die ersten zwölf Monate nach Inkrafttreten der Reform publiziert hat, abgeglichen.

8 Dr. Albrecht Walsleben von der Dokumentation des Bundesrates ist dafür zu danken, dass er mir diese Daten zur Verfügung gestellt hat.

9 Dagegen gelangt Marcus Höreth (2007: 727) in einer Untersuchung der verkündeten Gesetze seit Inkrafttreten der Föderalismusreform I bis einschließlich August $2007 \mathrm{zu}$ deutlich anderen Ergebnissen. Ihm zufolge sinkt der Anteil der zustimmungsbedürftigen Gesetze nur von $50 \%$ zwischen dem Beginn der 16. Legislaturperiode und dem Inkrafttreten der Reform auf 47,4 \% in den ersten zwölf Monaten nach der Reform. Allerdings ist Höreths Datengrundlage offensichtlich unvollständig, sodass seine Ergebnisse die Zustimmungsbedürftigkeit der Gesetze überschätzen (vgl. hierzu Zohlnhöfer 2008). 


\section{Abbildung 1}

Anteil der zustimmungsbedürftigen Gesetze an allen Gesetzen nach Legislaturperioden

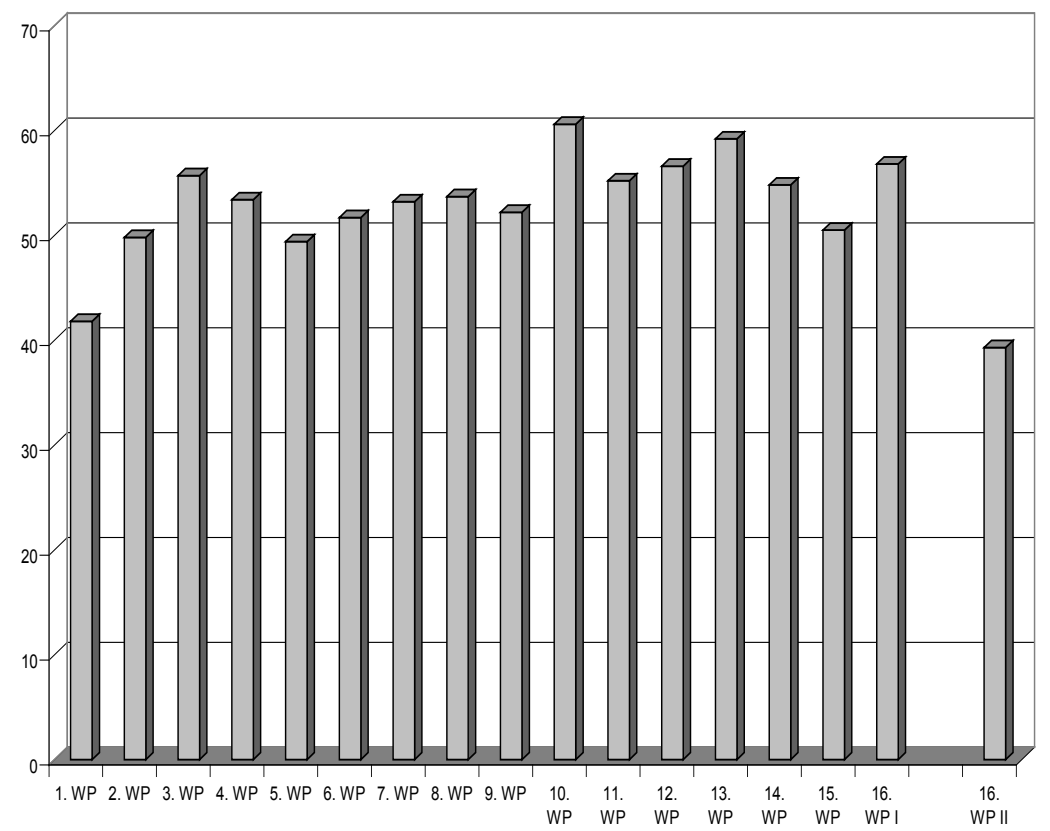

Quellen: Schindler 1999: 2430 f.; Feldkamp 2005: 578; Risse 2007: 709 (16. Wahlperiode bis zum Inkrafttreten der Reform); eigene Auswertung (16. Wahlperiode nach der Reform)

Ließen sich diese Zustimmungsquoten auf eine vollständige Wahlperiode hochrechnen, käme man in die Nähe der geringsten Zustimmungsquote in der Geschichte der Bundesrepublik (vgl. Abb. 1). Diese wurde bisher in der ersten Wahlperiode des Bundestags mit 41,8 \% erreicht - und diese Quote läge sogar noch höher als die Zustimmungsquote, die ich für die ersten 28 Monate seit Inkrafttreten der Föderalismusreform errechnet habe. Schaut man in die etwas jüngere Vergangenheit, wird noch deutlicher, dass die von mir berechneten Daten einen nennenswerten Effekt der Föderalismusreform auf die Zustimmungsbedürftigkeit von Bundesgesetzen plausibel machen. In den vier Legislaturperioden nach der Wiedervereinigung lagen die Zustimmungsquoten nämlich beispielsweise bei 56,6 \%, 59,2 \%, 54,8 \% und 
50,5 \% (Schindler 1999: 2430 f.; Feldkamp 2005: 578) und zwischen dem Beginn der 16. Legislaturperiode und dem Inkrafttreten der Föderalismusreform I lag sie bei 56,8 \% (Risse 2007: 709). ${ }^{10}$ Die Bundesregierung schließlich argumentiert sogar, dass die Quote zustimmungsbedürftiger Gesetze an allen Gesetzen im ersten Jahr nach Inkrafttreten der Reform nicht bei den tatsächlich erreichten $44,2 \%$, sondern bei $59,2 \%$ gelegen hätte, wenn die Reform nicht durchgesetzt worden wäre (BT-Drs. 16/8688: 42). All diese Daten deuten also darauf hin, dass die Föderalismusreform in der Tat zu einer Senkung der Zustimmungsquote um 10 bis 20 Prozentpunkte beigetragen haben könnte.

Mit derart hoch aggregierten Daten kann allerdings eine besonders wichtige Frage noch nicht beantwortet werden: Bringt diese Senkung auch tatsächlich eine höhere politische Handlungsfähigkeit der Bundesregierung mit sich? Die angeführten Zahlen könnten von Routineentscheidungen geprägt sein, die häufig unstrittig sind. Dann wäre zwar die Zustimmungsbedürftigkeit quantitativ zurückgegangen, aber der Handlungsspielraum der Bundesregierung wäre kaum gewachsen, weil die Gesetze, bei denen die Zustimmungsbedürftigkeit entfallen ist, ohnehin kaum vom Bundesrat beanstandet worden wären. Daher lohnt es, die 22 Gesetze etwas genauer zu analysieren, bei denen nach Angaben der Bundesregierung im ersten Jahr nach Inkrafttreten der Föderalismusreform I die Zustimmungsbedürftigkeit entfallen ist.

Bei diesem Schritt ist allerdings Vorsicht geboten: Es ist nämlich keineswegs unumstritten, wann ein Gesetz zustimmungsbedürftig ist. Zwischen 1972 und 2005 galt das beispielsweise bei gleichgerichteten Mehrheiten in Bundestag und Bundesrat in vier Prozent und bei gegenläufigen Mehrheiten sogar in acht Prozent der Fälle (Burkhart 2008: 187). Nun ist aber die Föderalismusreform I eines der besonders wichtigen Reformwerke der Großen Koalition, sodass die Bundesregierung ein Interesse daran hat, den Erfolg der Reform zu „beweisen“ (Höreth 2007: 720). Insofern ist es nicht unwahrscheinlich, dass die Bundesregierung die Zahl der Gesetze, die nach altem Recht der Zustimmung des Bundesrates bedurft hätten und bei denen folglich erst durch die Föderalismusreform die Zustimmungsbedürftigkeit entfallen ist, sehr hoch ansetzt. Es ist daher anzunehmen, dass eine Bundesregierung

10 Allerdings basieren die Zahlen von Risse, die sich auf alle vom Bundesrat im 2. Durchgang behandelten Gesetze beziehen, nicht auf der gleichen Datenbasis wie die von Schindler und Feldkamp sowie meine eigenen, die sich auf die verkündeten Gesetze beziehen. Die Verzerrung dürfte jedoch marginal sein. 
auch unter altem Recht vermutlich bei einem Teil dieser 22 Gesetze die Zustimmungsbedürftigkeit bestritten hätte, insbesondere, wenn sie mit einer Bundesratsmehrheit mit anderer parteipolitischer Couleur konfrontiert gewesen wäre. Marcus Höreth (2008: 145 ff.) meint sogar, dass bis zu elf der fraglichen 22 Gesetze auch nach altem Recht ohne Zustimmung des Bundesrates hätten verabschiedet werden können.

Wenn im Folgenden also diese 22 Gesetze behandelt werden, ist das die maximal mögliche Zahl von Gesetzen, bei denen durch die Föderalismusreform die Zustimmungsnotwendigkeit entfallen sein könnte. Doch wie konfliktträchtig war die Verabschiedung dieser 22 Gesetze (zum Folgenden vgl. Zohlnhöfer 2008: 418)? Der Bundesrat hat zu keinem einzigen dieser Vorhaben den Vermittlungsausschuss angerufen - d. h., auch wenn diese Gesetze zustimmungsbedürftig gewesen wären, wären sie womöglich nicht in die Fänge der Politikverflechtung geraten. Dies gilt umso mehr, als sich auch im Bundestag die Konfliktintensität in Grenzen hielt: So wurden drei dieser Gesetze einstimmig verabschiedet, bei fünf weiteren gab es immerhin keine Gegenstimmen und gegen weitere fünf dieser Gesetze stimmte nur die Linke. Das heißt, dass bei mehr als der Hälfte (13 von 22) der fraglichen Gesetze zwischen den etablierten Parteien schlicht kein Dissens bestand, während nur in zwei Fällen alle Oppositionsparteien gegen die Vorlage stimmten. Darüber hinaus gehörten die Materien, die mit den entsprechenden Entwürfen geregelt werden sollten, sicherlich nicht zu den zentralen Reformprojekten der Legislaturperiode. Das wird bereits daran deutlich, dass über ein Viertel der Vorlagen (sechs von 22) ohne Aussprache angenommen wurde, was in der Literatur als klarer Indikator gewertet wird, dass ,es sich um spezielle Anpassungsgesetze von marginaler Bedeutung" handelt (Ismayr 2008: 403). Dagegen kam es lediglich bei einem dieser Projekte, nämlich beim Vertragsarztrechtsänderungsgesetz, zu einer namentlichen Abstimmung, die als Indiz für eine überdurchschnittlich hohe Konfliktintensität interpretiert werden kann. Daneben waren noch das Terrorismusbekämpfungsergänzungsgesetz sowie das Passgesetz in nennenswertem Umfang - und auch innerhalb der Koalition - kontrovers (Burkhart 2008: 189), sodass auch die Verabschiedung dieser Gesetze durch die Reform vereinfacht worden sein sollte. Insgesamt zeigt sich aber, dass die schiere Zahl der nicht mehr zustimmungsbedürftigen Gesetze die Vergrößerung des Handlungsspielraums der Bundesregierung mit hoher Wahrscheinlichkeit überschätzt. 


\section{Die Schlüsselentscheidungen der rot-grünen Bundesregierung - eine kontrafaktische Analyse}

Die bisherige Diskussion zeigt, dass sich aus der Betrachtung der bisher existierenden Daten zwar bereits einige Trends ableiten lassen, dass die daraus zu gewinnenden Erkenntnisse jedoch noch nicht unbedingt verallgemeinerbar sind. Das gilt vor allem, weil der Untersuchungszeitraum seit Inkrafttreten der Föderalismusreform für empirische Analysen noch vergleichsweise kurz ist und die Daten daher durch Zufälligkeiten verzerrt sein könnten. Eine besonders wichtige Ursache für eine Verzerrung der Daten könnte darin bestehen, dass die Bundesregierung derzeit von einer Großen Koalition gebildet wird.

Aus zwei Gründen könnte die Große Koalition zu einer künstlich niedrigen Zahl von zustimmungsbedürftigen Gesetzen beitragen. Erstens besitzt sie eine eigene Mehrheit im Bundesrat, sodass parteipolitisch motivierte Konflikte zwischen Bundestag und Länderkammer unwahrscheinlich sind. Insofern dürfte die Zustimmung des Bundesrates in den meisten Fällen gesichert sein, sodass es auch nur in begrenztem Umfang zu Konflikten um die Frage kommt, ob ein Gesetz überhaupt zustimmungsbedürftig ist oder nicht. Hat der Bundesrat ohnehin vor, dem Gesetz zuzustimmen, wird er normalerweise kaum darauf bestehen, dass es zustimmungsbedürftig ist, wenn die Bundesregierung es als zustimmungsfrei einbringt. Anders könnte die Situation bei entgegengesetzten Mehrheiten zwischen Bundestag und Bundesrat sein, weil hier die Antwort auf die Frage, ob ein Gesetz zustimmungsbedürftig ist, darüber entscheidet, ob die Opposition das Gesetz qua Bundesrat beeinflussen kann oder nicht. Hier wird der Bundesrat also genau darüber wachen, dass die Zustimmungsbedürftigkeit eines Gesetzes auch tatsächlich festgestellt wird. Hinzu kommt das schon weiter oben vorgetragene Argument, wonach Bundes- wie Landesregierungen ein Interesse daran haben dürften, im Zweifelsfall die Zustimmungsbedürftigkeit eines Gesetzes zu verneinen, um durch eine gesunkene Quote zustimmungsbedürftiger Gesetze den Erfolg einer ihrer Kernreformen belegen zu können.

Deshalb werde ich im zweiten Schritt meiner Analyse die Schlüsselentscheidungen der rot-grünen Koalition zwischen 1998 und 2005 näher betrachten und kontrafaktisch analysieren, wie sich die Föderalismusreform auf die Politik der rot-grünen Bundesregierung ausgewirkt hätte, wenn die Reform bereits 1998 in Kraft getreten wäre, inwieweit sich also für diese Entscheidungen durch die Föderalismusreform die Zustimmungsbedürftigkeit 
verändert hätte. Diese Vorgehensweise bietet sich aus verschiedenen Gründen an: Erstens ermöglicht es die Konzentration auf Schlüsselentscheidungen, die Erweiterung der politischen Gestaltungsmöglichkeiten von Bundesregierungen jenseits von Routineentscheidungen $\mathrm{zu}$ analysieren. Zweitens konnte sich die rot-grüne Koalition nur in den ersten Monaten ihrer Regierungszeit auf eine eigene Mehrheit im Bundesrat verlassen, sodass sich am Beispiel der rot-grünen Koalition die Effekte von unterschiedlichen Mehrheiten in Bundestag und Bundesrat besser untersuchen lassen. Drittens schließlich liegen für sämtliche von der rot-grünen Regierung verabschiedeten Gesetze Daten über ihre Zustimmungsbedürftigkeit vor und nach der Reform vor, sodass sie also einer kontrafaktischen Analyse offenstehen.

Allerdings ist auch an dieser Stelle auf ein Datenproblem einzugehen. Die Daten für die kontrafaktische Analyse stammen nämlich aus der bereits angesprochenen Studie des Wissenschaftlichen Dienstes des Bundestages (Georgii/Borhanian 2006), deren Autoren auf der Basis dieser Daten zu dem Ergebnis gekommen waren, dass die Zustimmungsquote durch die Föderalismusreform auf rund $25 \%$ sinken könnte. $\mathrm{Zu}$ diesem Ergebnis gelangen sie, indem sie einerseits davon ausgehen, dass die Bundesregierung in allen Fällen des Artikels 84 Abs. 1 GG das Recht der Länder akzeptiert hätte, beim Gesetzesvollzug von den Regelungen des Bundes abzuweichen, und indem sie andererseits von einer sehr restriktiven Interpretation des neuen Art. 104a Abs. 4 ausgehen. Beide Annahmen sind mit hoher Wahrscheinlichkeit zu optimistisch (so schon Burkhart/Manow 2006), wie sich etwa daran zeigt, dass die Bundesregierung bisher bei rund zehn Prozent der Gesetzentwürfe auf einer einheitlichen Umsetzung, also der die Zustimmungsbedürftigkeit auslösenden Ausnahmeregelung des Art. 84 Abs.1 GG, bestand. ${ }^{11}$

Der neue Art. 104a Abs. 4 GG löste im ersten Jahr nach Inkrafttreten der Reform dagegen lediglich sieben Mal die Zustimmungsbedürftigkeit aus, davon lediglich in vier Fällen als alleiniger Auslöser für die Zustimmungs-

11 Nach den Daten der Bundesregierung für das erste Jahr nach Inkrafttreten der Reform waren 15 von 147 Gesetzen aufgrund des Art. 84 n. F. zustimmungsbedürftig (BT-Drs. 16/8688). Bei den Gesetzentwürfen, die im Bundesrat zwischen September 2006 und September 2007 im zweiten Durchgang behandelt wurden, waren es 18 von 166 Gesetzentwürfen (Risse 2007: 711). Meiner Auswertung der Daten der Dokumentation des Bundesrates zufolge, die einen längeren Zeitraum abdecken, war der Art. 84 n. F. in 25 von 300 Fällen einschlägig. 
bedürftigkeit. ${ }^{12}$ In keinem Fall wäre das entsprechende Gesetz nach Einschätzung der Bundesregierung vor der Reform zustimmungsfrei gewesen (eigene Auswertung der Anlage 1 in BT-Drs. 16/8688). Dennoch ist davon auszugehen, dass die zugrunde liegenden Daten die Senkung der Zustimmungsbedürftigkeit tendenziell überschätzen.

Was sind aber Schlüsselentscheidungen, die ja im Folgenden untersucht werden sollen? Nach Klaus von Beyme (1998) zeichnen sich Schlüsselentscheidungen durch drei Kriterien aus: a) durch den innovativen Charakter der Gesetze, b) durch die öffentliche Aufmerksamkeit des Themas in den Medien sowie in der Gesellschaft als Ganzes und c) durch eine hohe Konfliktintensität. Mithilfe der Geschäftsberichte der Bundesregierung, der Standardwerke zu den beiden Regierungen Schröder, einer Befragung einiger Rot-Grün-Experten sowie der Aufstellung von Schlüsselentscheidungen bei Reutter (2007: 305) wurden aus den 963 Gesetzen, die der Bundestag in der 14. und 15. Wahlperiode beschlossen hat, zwei Listen erstellt: eine mit den Entscheidungen, bei denen es sich zweifellos um Schlüsselentscheidungen handelt, sozusagen die Schlüsselentscheidungen im engeren Sinne, und eine zweite, in die auch Zweifelsfälle aufgenommen wurden. ${ }^{13}$

Bei diesem Vorgehen komme ich auf 24 Schlüsselentscheidungen im engeren Sinne - das entspricht etwa der Zahl von um die 10 Entscheidungen pro Legislaturperiode, die Klaus von Beyme für seine Studie über den Gesetzgeber zugrunde gelegt hat. Bei den Schlüsselentscheidungen im weiteren Sinne werden dagegen noch 45 zusätzliche Vorhaben berücksichtigt,

12 Nach den Daten der Dokumentation des Bundesrates löste Art. 104a Abs. 4 GG in den ersten 27 Monaten nach Inkrafttreten der Reform in 13 Fällen die Zustimmungsbedürftigkeit aus, davon acht Mal als alleiniger Auslöser (eigene Auswertung).

13 Eine Liste der untersuchten Schlüsselentscheidungen findet sich im Anhang. Meine Liste unterscheidet sich von Reutters (2007) Aufstellung darin, dass einerseits drei von Reutters Schlüsselentscheidungen, nämlich das 7. Hochschulrahmengesetzänderungsgesetz, das Gesetz zur Errichtung der Akademie der Künste sowie das Gesetz zur Errichtung einer Stiftung „Erinnerung, Verantwortung und Zukunft“ nicht aufgenommen wurden, weil es sich dabei $\mathrm{m}$. E. nicht um weitreichende Richtungsentscheidungen handelte. Die Aufnahme dieser Gesetze würde die im Folgenden präsentierten Ergebnisse allerdings ohnehin nicht ändern. Andererseits wurde eine ganze Reihe von Gesetzen, die bei Reutter fehlen, mir aber wichtig erscheinen, aufgenommen, so beispielsweise das 5. und das 6. HRGÄndG, das Bundeswehrneuausrichtungsgesetz, das Parlamentsbeteiligungsgesetz, das Gesetz zu dem Vertrag vom 29. Oktober 2004 über eine Verfassung für Europa, das Erneuerbare-Energien-Gesetz, das Gesetz zur geordneten Beendigung der Kernenergienutzung zur gewerblichen Erzeugung von Elektrizität und das Treibhausgas-Emissionshandelsgesetz, um nur einige zu nennen. 
sodass ich auf insgesamt 69 Entscheidungen für die zwei Legislaturperioden zusammen komme.

Ein erster interessanter Befund betrifft die Frage, ob der Bund die fraglichen Entscheidungen nach Inkrafttreten der Reform überhaupt noch hätte treffen dürfen oder ob einige in Bereiche fallen, die nach der Reform in den Bereich der ausschließlichen Gesetzgebung der Länder fallen. Letzteres kommt in der Tat vor. Zwei der 24 Schlüsselentscheidungen im engeren Sinne, nämlich das 5. und das 6. Gesetz zur Änderung des Hochschulrahmengesetzes, könnte der Bund heute nicht mehr erlassen. Zwar scheiterten beide Gesetze auch schon vor Inkrafttreten der Reform, weil das Bundesverfassungsgericht jeweils eine Überschreitung der Gesetzgebungskompetenz des Bundes monierte. Gleichzeitig beließen die Richter dem Bund seinerzeit aber noch bestimmte Kompetenzen, die es beispielsweise ermöglichten, die mit dem 5. HRGÄndG zunächst gescheiterte Einführung von Juniorprofessuren doch noch durchzusetzen, nämlich mit dem Gesetz zur Änderung dienst- und arbeitsrechtlicher Vorschriften im Hochschulbereich von 2004 (vgl. Kneip 2007: 229 ff.; Wolf/ Henkes 2007: 362 ff.).

Nach der Föderalismusreform wäre dies nicht mehr möglich gewesen. Entsprechend entfiel auch die Gesetzgebungskompetenz des Bundes für das gerade angesprochene Gesetz zur Änderung dienst- und arbeitsrechtlicher Vorschriften im Hochschulbereich weitgehend, das ich als Schlüsselentscheidung im weiteren Sinne klassifiziert habe. Da schließlich auch die Gesetzgebungskompetenz des Bundes zum Professorenbesoldungsgesetz und zum größten Teil auch zum Lebenspartnerschaftgesetzergänzungsgesetz entfallen sind, komme ich für die 69 Schlüsselentscheidungen im weiteren Sinne auf fünf Gesetze, die der Bund heute gar nicht mehr oder weitgehend nicht mehr erlassen könnte, wobei - wenig überraschend - die meisten von ihnen den Bereich der Hochschulpolitik betreffen.

Von den verbleibenden 22 Schlüsselentscheidungen im engeren Sinne waren vor der Reform 13 zustimmungsbedürftig, während neun dieser Entscheidungen auch gegen den Einspruch des Bundesrates durchgesetzt werden konnten. Damit ergibt sich für dieses Sample eine Zustimmungsquote vor der Reform von 59,1\%, die insofern leicht über der Zustimmungsquote aller Gesetze der rot-grünen Regierung liegt (53,5 \%). Nach der Reform wären dagegen nur noch zehn dieser Gesetze zustimmungsbedürftig gewesen, während nun zwölf auch gegen den Einspruch des Bundesrates hätten durchgesetzt werden können. Die Zustimmungsquote wäre demnach auf 45,5 \% gesunken. 
Bei welchen Gesetzen wäre nun aber die Zustimmungsbedürftigkeit entfallen? Dies sind das Gesetz zur Reform des Staatsangehörigkeitsrechts, das Gesundheitsreformgesetz 2000, das Erneuerbare-Energien-Gesetz sowie das GKV-Modernisierungsgesetz von 2003. Gleichzeitig wäre aber eine der rot-grünen Schlüsselentscheidungen, die seinerzeit ohne Zustimmung des Bundesrates durchgesetzt werden konnte, heute nach Art. 104a Abs. 4 GG zustimmungsbedürftig, nämlich das Tagesbetreuungsausbaugesetz.

Wie sieht es bei den Schlüsselentscheidungen im weiteren Sinne mit der Veränderung der Zustimmungsbedürftigkeit aus? Von den 64 einschlägigen Entscheidungen, die der Bund auch nach neuem Recht noch verabschieden könnte, waren vor der Reform 32 zustimmungsbedürftig, während 32 ohne Zustimmung des Bundesrates verabschiedet werden konnten. Die Zustimmungsquote lag also bei $50 \%$, und damit leicht unter dem Durchschnitt bei allen rot-grünen Gesetzen. Nach der Reform hätten noch 24 dieser Gesetze der Zustimmung des Bundesrates bedurft, 40 Gesetze hätten als Einspruchsgesetze verhandelt werden können, die Zustimmungsquote wäre also auf $37,5 \%$ gefallen.

Neben den schon angesprochenen Gesetzen wäre bei diesem größeren Sample auch die Zustimmungsbedürftigkeit beim BSE-Maßnahmengesetz, beim Zwölften SGB V-Änderungsgesetz, beim Gesetz zur Reform des Wohnungsbaurechts, beim Gesetz zur Bekämpfung des internationalen Terrorismus, beim Verbraucherinformationsgesetz, beim GKV-Solidaritätsstärkungsgesetz sowie bei der Einführung der Autobahnmaut entfallen, während das Europäisches Haftbefehlsgesetz und möglicherweise auch das Hochwasserschutzgesetz zustimmungsbedürftig geworden wären.

Insofern lässt also auch die kontrafaktische Analyse der rot-grünen Schlüsselentscheidungen die Erwartung entstehen, dass es durch die Föderalismusreform zu einer spürbaren Reduzierung der Zustimmungsbedürftigkeit auch bei Schlüsselentscheidungen gekommen sein könnte. Das gilt allerdings in erster Linie dann, wenn die Bundesregierung tatsächlich bereit wäre, Abweichungen beim Gesetzesvollzug zu akzeptieren. Da das nicht immer der Fall sein dürfte, sind die hier präsentierten Zahlen also der Tendenz nach etwas zu optimistisch, doch der Trend erscheint durchaus stabil.

Gleichwohl stellen sich noch zwei Fragen zur veränderten Zustimmungsbedürftigkeit bei den rot-grünen Schlüsselentscheidungen: Wie verteilt sich der Rückgang der Zustimmungsbedürftigkeit auf die einzelnen Politikfelder? Und wie konfliktträchtig waren die Gesetze, bei denen die Zustimmungsbedürftigkeit entfallen ist? 
Tabelle 1

Zahl der zustimmungsbedürftigen Schlüsselentscheidungen der rot-grünen Regierung nach Politikfeldern

\begin{tabular}{|l|c|c|}
\hline & vorher & nachher \\
\hline Arbeitsmarkt & 3 & 3 \\
\hline Äußeres/Verteidigung/Europa & 2 & 2 \\
\hline Bau/Verkehr & 2 & 0 \\
\hline Finanzen & 8 & 8 \\
\hline Gesundheit & 4 & 0 \\
\hline Inneres und Justiz & 4 & 3 \\
\hline Landwirtschaft u. Verbraucherschutz & 2 & 0 \\
\hline Soziales & 4 & 4 \\
\hline Umwelt & 2 & 2 \\
\hline
\end{tabular}

Quelle: eigene Auswertung; Zustimmungsbedürftigkeit vor und nach der Reform nach Georgii/ Borhanian 2006.

Ein Blick auf die Politikfelder zeigt in der Tat deutliche Unterschiede (vgl. Tabelle 1). Besonders die Finanzpolitik ist von Veränderungen weitgehend ausgenommen geblieben: Von acht finanzpolitischen Schlüsselentscheidungen, die vor der Reform der Zustimmung durch den Bundesrat bedurften, entfiel diese Notwendigkeit bei keiner einzigen. Auch in der Außen- und der Arbeitsmarkt- sowie der Sozialpolitik entfiel bei keiner einzigen Schlüsselentscheidung die Zustimmungsnotwendigkeit, doch ist dort der Anteil zustimmungsbedürftiger Gesetze insgesamt deutlich geringer als in der Finanzpolitik. Dagegen schlagen sich die Effekte der Föderalismusreform in der Innen- und Rechtspolitik durchaus nieder, wo bei zwei von vier vormals zustimmungsbedürftigen Gesetzen die Zustimmungsbedürftigkeit entfallen wäre, wenngleich bei einem Gesetz auch eine neue Zustimmungsnotwendigkeit entstanden wäre. Noch deutlichere Effekte finden sich in den Politikfeldern Landwirtschaft und Verbraucherschutz sowie Bau und Verkehr, wo bei allen vorher zustimmungsbedürftigen Gesetzen die Zustimmungsbedürftigkeit entfallen wäre. Gleiches gilt für die Gesundheitspolitik, wären doch alle vier betrachteten Gesundheitsreformen, die seinerzeit der Zustimmung des Bundesrates bedurften, heute lediglich Einspruchsgesetze. Allerdings 
ist gerade bei der Gesundheitspolitik vor allzu viel Hoffnung zu warnen, stellte sich die - nach Inkrafttreten der Föderalismusreform verabschiedete Gesundheitsreform der Großen Koalition doch neuerlich als zustimmungsbedürftig heraus.

Doch wie konfliktträchtig waren eigentlich die Gesetze, bei denen die Zustimmungsbedürftigkeit entfallen ist? Die Antwort auf diese Frage kann Hinweise darauf geben, inwieweit Bundesregierungen durch die Föderalismusreform tatsächlich politischen Handlungsspielraum gewonnen haben.

Wenn man analysiert, zu welchem der elf Gesetze, bei denen wegen der Föderalismusreform die Zustimmungsbedürftigkeit heute nicht mehr bestehen würde, der Bundesrat den Vermittlungsausschuss angerufen hat, ist zunächst auf die Sonderstellung des GKV-Solidaritätsstärkungsgesetzes hinzuweisen, das nämlich noch unter den Bedingungen gleichgerichteter Mehrheiten in Bundestag und Bundesrat verabschiedet wurde, sodass die Nicht-Anrufung des Vermittlungsausschusses wohl auf die gleichgerichteten Mehrheitsverhältnisse zurückgeführt werden kann. Von den verbleibenden zehn Gesetzen wurde zu vieren der Vermittlungsausschuss angerufen. Dabei scheiterte das Vermittlungsverfahren im Falle des Verbraucherinformationsgesetzes sogar zweimal gänzlich, sodass das Gesetz nicht in Kraft treten konnte, während das Gesundheitsreformgesetz 2000 nach einem erfolgreichen Vermittlungsverfahren ebenso in modifizierter Fassung in Kraft treten konnte wie das Autobahnmautgesetz und das Zwölfte SGB V-Änderungsgesetz.

$\mathrm{Zu}$ diesen vier Fällen sind allerdings in jedem Fall noch die Reform des Staatsangehörigkeitsrechts von 1999 sowie das GKV-Modernisierungsgesetz von 2003 hinzuzurechnen. In beiden Fällen kam es zwar nicht zu einem formalen Vermittlungsverfahren, doch beide Male erfolgte die Ausarbeitung der Gesetze unter Beteiligung der Parteien und Landesregierungen, deren Zustimmung im Bundesrat notwendig war. Im Falle der Reform des Staatsangehörigkeitsrechts benötigte die rot-grüne Regierung im Bundesrat die Zustimmung des sozial-liberal regierten Rheinland-Pfalz, sodass eine Reihe von Konzessionen an die FDP gemacht wurde, insbesondere hinsichtlich des Verzichts auf die Regelhinnahme der doppelten Staatsbürgerschaft (Busch 2003: 312 f.). Bei der Ausarbeitung des GKV-Modernisierungsgesetzes wurde in gleicher Weise von Anfang an auf die Einbindung der Unionsparteien geachtet, was sich in einer Reihe von Regelungen niederschlug, die die rot-grüne Regierung zweifellos nicht eingeführt hätte, wenn sie keine Rücksicht auf den Bundesrat zu nehmen gehabt hätte (Bandelow/Hartmann 2007: 338 ff.). Das betrifft insbesondere die Erhöhung der Zuzahlungen und die 
Einführung der Praxisgebühr. Zumindest die genannten sechs Schlüsselentscheidungen wären also vermutlich in erkennbar anderer, stärker den Präferenzen der Regierung entsprechender Form verabschiedet worden, wenn die Föderalismusreform bereits 1998 in Kraft gewesen wäre.

Doch auch die übrigen Gesetze, die durch die Föderalismusreform zu Einspruchgesetzen geworden wären, wiesen ein vergleichsweise hohes Maß an Konflikthaftigkeit auf, wie sich daran zeigt, dass sie im Bundestag in der Regel gegen die Stimmen der Hauptoppositionsfraktion verabschiedet wurden (vgl. Tabelle 2). Diese Ergebnisse sprechen dafür, dass in all diesen Fällen der politische Handlungsspielraum der Bundesregierung in der Tat etwas gewachsen ist.

Allerdings bliebe die Betrachtung unvollständig, wenn man nicht auch die Gesetze in die Analyse einschließen würde, bei denen durch die Föderalismusreform überhaupt erst eine Zustimmungsbedürftigkeit geschaffen wurde. Das betrifft das Tagesbetreuungsausbaugesetz, das Europäische Haftbefehlsgesetz sowie vermutlich das Hochwasserschutzgesetz (vgl. Tabelle 2). In allen drei Fällen wurde interessanterweise in der Tat der Vermittlungsausschuss angerufen. Beim Tagesbetreuungsausbaugesetz musste das Vermittlungsverfahren sogar ohne Einigungsvorschlag abgeschlossen werden (BRat Drs. 986/04) und auch beim Europäischen Haftbefehlsgesetz kam es nur zu einem unechten Einigungsvorschlag, nämlich der Bestätigung des vom Bundestag beschlossenen Gesetzes (BRat Drs. 451/04). Dies war seinerzeit insoweit unproblematisch, als beide Gesetze auch gegen den Bundesrat durchgesetzt werden konnten und durchgesetzt wurden (BT PIPr. 15/149, 17.12.2004, S. 14033; PIPr. 15/113, 16.6.2004, S. 10309-10311). Dagegen wären bei diesen Gesetzen in Zukunft erhebliche Konzessionen notwendig, um den Bundesrat zur Zustimmung zu bewegen. Insofern ist der Nettoeffekt der Föderalismusreform auf die politische Gestaltungsfähigkeit der Bundesregierung zwar immer noch positiv, aber doch geringer, als er ohne die Änderung des Art. 104a Abs. 4 GG hätte sein können.

Die Föderalismusreform könnte also, so lassen sich die ersten beiden Schritte meiner Analyse zusammenfassen, tatsächlich zu einer gewissen Entflechtung bei der Gesetzgebung beigetragen haben, wenngleich diese Entflechtung nach Politikfeldern variiert und insgesamt bei weitem nicht so weit reicht, wie das die Rede von der „Mutter aller Reformen“ erwarten ließe. 
Tabelle 2

Gesetze, bei denen sich Zustimmungsbedürftigkeit verändert hätte, und Konfliktintensität

\begin{tabular}{|c|c|c|c|c|}
\hline Gesetz & $\begin{array}{l}\text { Anrufung } \\
\text { VA? }\end{array}$ & $\begin{array}{l}\text { Ein- } \\
\text { stimmig? }\end{array}$ & $\begin{array}{l}\text { Große } \\
\text { Mehr- } \\
\text { heit? }\end{array}$ & $\begin{array}{l}\text { Namentl. } \\
\text { Abstim- } \\
\text { mung? }\end{array}$ \\
\hline \multicolumn{5}{|l|}{ Neu Zustimmungsfrei } \\
\hline Staatsangehörigkeit & Nein & Nein & $\begin{array}{l}\text { Koalition } \\
+ \text { FDP + } \\
\text { meiste } \\
\text { PDS }\end{array}$ & $\mathrm{Ja}$ \\
\hline Erneuerbare-Energien-Gesetz & Nein & Nein & $\begin{array}{l}\text { Koalition } \\
+ \text { PDS + } \\
\text { Teile FDP }\end{array}$ & $\mathrm{Ja}$ \\
\hline Gesundheitsreform 2000 & $\mathrm{Ja}$ & Nein & Nein & $\mathrm{Ja}$ \\
\hline GKV-Modernisierungsgesetz & Nein & Nein & $\begin{array}{l}\text { Koalition } \\
+\mathrm{CDU} / \\
\mathrm{CSU}\end{array}$ & $\mathrm{Ja}$ \\
\hline $\begin{array}{l}\text { Reform des Wohnungsbau- } \\
\text { rechts }\end{array}$ & Nein & Nein & Nein & Nein \\
\hline $\begin{array}{l}\text { Gesetz zur Bekämpfung des } \\
\text { internationalen Terrorismus }\end{array}$ & Nein & Nein & $\begin{array}{l}\text { Koalition } \\
+\mathrm{CDU} / \\
\mathrm{CSU} \\
\end{array}$ & Nein \\
\hline Verbraucherinformationsgesetz & \begin{tabular}{|l} 
Ja (keine \\
Einig.) \\
\end{tabular} & Nein & $\begin{array}{l}\text { Koalition } \\
+ \text { PDS }\end{array}$ & Nein \\
\hline BSE-Maßnahmengesetz & Nein & Nein & $\begin{array}{l}\text { Koalition } \\
+\mathrm{CDU} / \\
\text { CSU } \\
\text { (weniger } 1 \\
\text { Abg.) }\end{array}$ & Nein \\
\hline 12. SGB V-Änderungsgesetz & $\mathrm{Ja}(2 \mathrm{x})$ & Nein & Nein & Nein \\
\hline Autobahnmaut & $\mathrm{Ja}$ & k. A. & k. A. & Nein \\
\hline $\begin{array}{l}\text { GKV-Solidaritätsstärkungs- } \\
\text { gesetz }\end{array}$ & Nein & Nein & Nein & Nein \\
\hline
\end{tabular}




\begin{tabular}{|l|l|l|l|l|}
\hline Gesetz & $\begin{array}{l}\text { Anrufung } \\
\text { VA? }\end{array}$ & $\begin{array}{l}\text { Ein- } \\
\text { stimmig? }\end{array}$ & $\begin{array}{l}\text { Große } \\
\text { Mehr- } \\
\text { heit? }\end{array}$ & $\begin{array}{l}\text { Namentl. } \\
\text { Abstim- } \\
\text { mung? }\end{array}$ \\
\hline Neu Zustimmungsbedürftig & \multicolumn{3}{|l|}{} \\
\hline Tagesbetreunngsausbaugesetz & Ja & Nein & $\begin{array}{l}\text { Keine } \\
\text { (bzw. 3) } \\
\text { Gegen- } \\
\text { stimmen; } \\
\text { Enthaltung } \\
\text { CDU/CSU } \\
\text { + FDP }\end{array}$ & $\begin{array}{l}\text { Ja } \\
\text { (Zurück- } \\
\text { weisung } \\
\text { Ein- } \\
\text { spruch } \\
\text { BRat) }\end{array}$ \\
\hline Europäisches Haftbefehlsgesetz & Ja & $\begin{array}{l}\text { Ja (Nein: } \\
\text { Vermitt- } \\
\text { lungser- } \\
\text { gebnis) }\end{array}$ & $\begin{array}{l}\text { Ja (Nein: } \\
\text { Vermitt- } \\
\text { lungser- } \\
\text { gebnis) }\end{array}$ & $\begin{array}{l}\text { Ja } \\
\text { (Zurück- } \\
\text { weisung } \\
\text { Ein- } \\
\text { spruch } \\
\text { BRat) }\end{array}$ \\
\hline $\begin{array}{l}\text { Hochwasserschutzgesetz } \\
\text { (unklar) }\end{array}$ & Ja & $\begin{array}{l}\text { Nein (Ja: } \\
\text { Vermitt- }\end{array}$ & $\begin{array}{l}\text { Nein (Ja: } \\
\text { Vermitt- } \\
\text { lungser- } \\
\text { gebnis) }\end{array}$ & $\begin{array}{l}\text { Nein } \\
\text { lungser- } \\
\text { gebnis) }\end{array}$ \\
\hline
\end{tabular}

Quelle: eigene Auswertung der Datenbank „Stand der Gesetzgebung“; kursiv: Schlüsselentscheidungen im engeren Sinne

\section{Stimmen die Annahmen? Föderalismusreform und der politische Prozess}

Im dritten Schritt meiner Analyse will ich mich von der strikten, sozusagen rein „technischen“ Gesetzgebungsanalyse lösen, die sich auf die Untersuchung der Zustimmungsbedürftigkeit von Gesetzen konzentriert. Vielmehr soll der gesamte Willensbildungsprozess in den Blick genommen, und insofern die legislative Politikverflechtung kontextualisiert werden.

Vielfach wird angenommen, dass der seit den 90er-Jahren diagnostizierte Reformstau - verstanden als Unfähigkeit von Regierungen, kohärente oder weitreichende Reformen zu verabschieden - in erster Linie durch die Politikverflechtung verursacht wurde. Die Schlussfolgerung lautet also, dass eine Reform des föderalen Systems die Lösung für das Problem des Reformstaus sein müsste. Eine nähere Betrachtung der deutschen Willensbildungs- 
prozesse zeigt jedoch, dass diese Annahme nicht korrekt ist, jedenfalls nicht vollständig. ${ }^{14}$

Nimmt man wiederum die Schlüsselentscheidungen der rot-grünen Koalition als Beispiel, wird deutlich, dass der Einfluss des Bundesrates die Inkohärenz der Reformen der Regierung Schröder aus mindestens drei Gründen nicht vollständig erklären kann: Erstens war die Regierung Schröder relativ erfolgreich darin, den Bundesrat zu umgehen und so die wichtigsten Teile ihrer Reformen durchzusetzen (vgl. zum Folgenden Zohlnhöfer 2003: 411 f.). Ein altbekannter Weg, wichtige Teile umstrittener Reformen gegen den Bundesrat durchzusetzen, besteht in der Aufspaltung der Gesetze in zustimmungsbedürftige und zustimmungsfreie Teile. Dies erlaubte es der Regierung häufig, die wichtigsten Teile ihrer Reformen auch ohne eine Einigung mit der Länderkammer durchzusetzen. Die rot-grüne Koalition beschritt diesen Weg bei der Rentenreform der ersten Wahlperiode, beim Lebenspartnerschaftsgesetz, bei der Gesundheitsreform 2000, beim Sparpaket von 1999, beim 5. Gesetz zur Änderung des Hochschulrahmengesetzes, von dem das Gesetz zur Reform der Professorenbesoldung abgespalten wurde, aber auch bei den Hartz-Gesetzen und beim Beitragssatzsicherungsgesetz. Ähnliche, wenngleich technisch etwas abweichende gesetzgebungstechnische Umwege wurden beim Kraft-Wärme-Kopplungsgesetz genommen.

Ein anderer Weg zur Umgehung der Vetomacht des Bundesrates ließ sich zumindest solange beschreiten, wie Regierungen ,gemischter“ Länder im Bundesrat den Ausschlag gaben, also die Regierungen solcher Länder, die von Koalitionen regiert werden, deren einer Partner zur Regierungskoalition auf Bundesebene gehört, während der andere auf Bundesebene in der Opposition ist. Bei der Steuerreform 2000 sowie beim zustimmungsbedürftigen Teil der Riester-Rentenreform wurde die Zustimmung einiger gemischt regierter Länder durch finanzielle Anreize ohne direkten Bezug zur jeweiligen Reform im wahrsten Sinne des Wortes erkauft. Solche „Kaufstrategien“ erlaubten es der rot-grünen Regierung, die im Bundesrat entscheidenden „gemischt“ regierten Bundesländer zur Zustimmung zu bewegen, ohne zusätzliche inhaltliche Konzessionen machen zu müssen.

14 Benz (2005: 209 f.) zufolge verbaute die Annahme, eine Entflechtung sei nötig, auch die Chance, in der ersten Föderalismuskommission alternative Flexibilisierungsoptionen ernsthaft zu prüfen. Diese These wird hier nicht weiter verfolgt, weil auch diese alternativen Flexibilisierungsoptionen ja an der Reform des föderalen Systems ansetzten und die Bedeutung des Parteienwettbewerbs insofern kaum thematisierten. 
Zweitens hat der Bundesrat in einer bemerkenswerten Anzahl von Fällen Reformen, insbesondere in der Wirtschafts- und Sozialpolitik, nicht abgeschwächt, sondern eher weiterreichende Reformen ermöglicht. Ein Beispiel ist der Spitzensteuersatz der Einkommensteuer, den die Bundesregierung ursprünglich lediglich auf $45 \%$ reduzieren wollte und der schließlich auf Druck des Bundesrates auf $42 \%$ gesenkt wurde. Ebenso hätten viele der Reformen der „Agenda 2010“ ohne die christlich-liberale Mehrheit im Bundesrat nicht verabschiedet werden können, weil die SPD-Fraktion im Bundestag teilweise nicht bereit war, in die von der Regierung gewünschte liberale Richtung zu gehen. Die zweite rot-grüne Gesundheitsreform und vor allem die vier Hartz-Reformen können als einschlägige Beispiele dienen (für Details vgl. Zohlnhöfer/Egle 2007: 14 f.).

Drittens schließlich erstreckt sich das inkohärente Reformmuster nicht nur auf die zustimmungsbedürftige Gesetzgebung. In weiten Teilen der Arbeitsmarktgesetzgebung, der Rentenpolitik sowie bei der Ökosteuerreform besaß der Bundesrat kein Vetorecht. Dennoch blieben auch in diesen Bereichen kohärente Reformen aus.

Was ist also dann verantwortlich für den Reformstau? Meine Untersuchungen zu den Willensbildungsprozessen der Regierungen Kohl und Schröder deuten darauf hin, dass der Wettbewerb um Wählerstimmen ein wichtiger Schlüssel zum Verständnis der deutschen Politik ist.

Wie folgenreich ein Festhalten an heftig umstrittenen Reformen gerade in der Bundesrepublik ist, die sich durch die Vielzahl von Landtagswahlen geradezu in einem Dauerwahlkampf befindet, konnte die rot-grüne Regierung bereits am Anfang ihrer Regierungstätigkeit am Fall der Neuregelung des Staatsbürgerschaftsrechts feststellen (dazu Busch 2003: 311 ff.). Die Unionsparteien initiierten eine Unterschriftenaktion gegen die doppelte Staatsbürgerschaft und machten diese Frage zum Thema des hessischen Landtagswahlkampfes 1999, der zum Regierungswechsel in Wiesbaden, zum Verlust der rot-grünen Mehrheit im Bundesrat und damit letztlich zu einer moderateren Fassung der Reform führte. Die Verabschiedung eines Zuwanderungsgesetzes, die vor der Übernahme der Regierung als vordringlich versprochen worden war, wurde vor diesem Hintergrund dann zunächst zurückgestellt (Busch 2003: 313).

Ein halbes Jahr später erlitt die SPD dann nach der Ankündigung eines Sparpakets empfindliche Niederlagen bei einer Reihe von Landtagswahlen, die zumindest teilweise auf die in dem Sparpaket enthaltenen Sozialkürzungen zurückgeführt wurden. Entsprechend schreckte insbesondere die SPD 
anschließend vor weiteren Ausgabenkürzungen zurück und verschob die geplante Gesundheitsreform in die nächste Wahlperiode (Zohlnhöfer 2004: 394 f.).

In anderen Bereichen wurden Reformmaßnahmen erst gar nicht oder nur in sehr moderater Form verabschiedet, um nicht den Unwillen der Wähler auf sich zu ziehen. Das gilt etwa für die Arbeitsmarktpolitik, in der die Regierung in ihrer ersten Amtszeit so lange auf Reformen verzichtete, wie eine Senkung der Arbeitslosenzahlen auf das anvisierte Ziel von 3,5 Mio. allein aufgrund der demografischen Entwicklung erhofft werden konnte (Blancke/ Schmid 2003: 225). Und das gilt für die Ökosteuern, die weit weniger stark erhöht wurden, als nötig gewesen wäre, um das selbst gesteckte Ziel bei der Reduzierung der Rentenbeiträge zu erreichen. Zusätzlich kam es bei der Ökosteuerreform angesichts von Protestaktionen in mehreren Städten zu sogenannten „sozialen Korrekturen“, die die angestrebten Steuerungsleistungen der Ökosteuer allerdings erheblich infrage stellten (Raschke 2001: 217 ff., 230). Zudem ist diese Orientierung am Wahlterminkalender und an den reformaversen Impulsen der Wähler keineswegs auf die rot-grüne Regierung beschränkt, wie beispielhaft ein Blick auf die Wirtschafts- und die Sozialpolitik der Regierung Kohl zeigt (Zohlnhöfer 2001; Schmidt 2005).

Wenn dieses Argument aber insgesamt zutrifft, dann heißt das nichts anderes, als dass das Ziel kohärenter Reformpolitik selbst dann kaum zu erreichen ist, wenn die Föderalismusreform I denn tatsächlich wenigstens in ersten Ansätzen zu einer Politikentflechtung beigetragen haben sollte.

\section{Fazit: Der Politikverflechtungsfalle entwischt?}

Die Reform des föderalen Systems der Bundesrepublik hat erstens den Handlungsspielraum der Länder erhöht, wenngleich in begrenztem Umfang, weil die Gesetzgebungskompetenzen der Länder weiterhin begrenzt sind und die Länder sich vorläufig noch häufig in die horizontale Koordinierung flüchten.

Zweitens wurde bei der quantitativen Betrachtung der ersten 28 Monate nach Inkrafttreten der Reform ebenso wie bei der kontrafaktischen Betrachtung der Schlüsselentscheidungen der rot-grünen Bundesregierung deutlich, dass sich die Zustimmungsbedürftigkeit von Bundesgesetzen doch in nennenswertem Umfang verringert hat, allerdings bei erheblichen politikfeldspezifischen Unterschieden. Zudem zeigte sich bei den Gesetzen der Großen Koalition, bei denen die Zustimmungsbedürftigkeit entfallen ist, dass diese fast durchgängig kaum umstritten und häufig von marginaler Bedeutung waren; 
dagegen ergab die kontrafaktische Untersuchung der rot-grünen Gesetzgebung, dass auch einige hoch umstrittene Gesetze vom Wegfall der $\mathrm{Zu}$ stimmungsbedürftigkeit betroffen sein könnten.

Ist also mit der Föderalismusreform I ein Schritt aus der Politikverflechtung gelungen, haben die Akteure die Politikverflechtungsfalle ausgetrickst? Scharpf (1985) hatte ja argumentiert, dass in politikverflochtenen Systemen nicht nur systematisch suboptimale Entscheidungen zu erwarten seien. Vielmehr ließen sich die institutionellen Strukturen der Politikverflechtung selbst, die diese ineffizienten Entscheidungen erzeugen, auch dann kaum ändern, wenn alle Beteiligten mit den Ergebnissen unzufrieden seien. Die hier vorgestellten Ergebnisse scheinen wenigstens auf den ersten Blick darauf hinzudeuten, dass die politischen Akteure mit der Föderalismusreform I in der Tat der Politikverflechtungsfalle entwischt sind, es ihnen also gelungen ist, eine institutionelle Reform vorzunehmen, die die blockadeanfällige deutsche Gesetzgebung wenigstens etwas entflochten hat.

Allerdings wäre es voreilig, die Theorie der Politikverflechtung als erledigt zu betrachten. Gerade hinsichtlich der Entwicklung der Zustimmungsquote sind die Ergebnisse vorläufiger Natur. Dies ist nicht zuletzt der Tatsache geschuldet, dass wir es derzeit mit einer Großen Koalition zu tun haben, die auch die Mehrheit im Bundesrat kontrolliert. Der Lackmustest für die Wirkung der Artikel 84 Abs. 1 und 104a Abs. 4 GG auf die Zustimmungsbedürftigkeit von Bundesgesetzen steht noch aus, und wird erst dann kommen, wenn eine Bundesregierung mit einer parteipolitisch gegenläufigen Mehrheit im Bundesrat konfrontiert ist. Dabei wird es dann einerseits darauf ankommen, ob die Bundesregierung auch bei kontroversen Projekten von der Abweichungsoption des Artikel 84 Absatz 1 GG Gebrauch machen wird. Diese Option birgt nämlich potenziell das Risiko, dass das Gesetz seine Ziele verfehlt, wenn einige (oder sogar eine Mehrheit der) Länder es nicht auf die von der Bundesregierung gewünschte Art ausführen. Wenn die Bundesregierung also befürchtet, dass die Länder eine Reform über den Vollzug aushebeln, wird sie wahrscheinlich eine einheitliche Ausführung sicherstellen wollen, was wiederum die Zustimmung des Bundesrates nötig macht (vgl. Scharpf 2006: 2 f.).

Andererseits wird es spätestens bei gegenläufigen Mehrheiten in Bundestag und Bundesrat darauf ankommen, wie eng oder weit das Bundesverfassungsgericht den neuen Artikel 104a Absatz 4 GG auslegt, der in hohem Maße interpretationsbedürftig ist, und zwar aus mindestens drei Gründen (vgl. zum Folgenden Meyer 2006: 6-10; Scharpf 2006: 3 ff.): Erstens ist 
unklar, was alles als „,vergleichbare Dienstleistungen“ zu verstehen ist. Zweitens wird das Verfassungsgericht darüber zu entscheiden haben, ob die $\mathrm{Zu}$ stimmung des Bundesrates lediglich bei ,erheblichen Kostenbelastungen der Länder" entsteht. So steht es zwar in der Gesetzesbegründung (BT-Drs. 16/813: 18), aber eben nicht im Grundgesetz selbst. Und drittens ist unklar, ob der Bund sich von der Zustimmungsbedürftigkeit bei Gesetzen, die geldwerte Sachleistungen oder vergleichbare Dienstleistungen gewähren, freikaufen kann. Der Konditionalsatz, dass die Zustimmungserfordernis nur entsteht, ,wenn daraus entstehende Ausgaben von den Ländern zu tragen sind", der erst bei der parlamentarischen Beratung der Föderalismusreform eingefügt wurde (BT-Drs. 16/2010: 6 f.), deutet darauf hin, dass diese Möglichkeit existiert. Auf der anderen Seite ermächtigt Art. 104a Abs. 3 den Bund aber explizit nur für Geldleistungsgesetze, nicht aber für Gesetze, die geldwerte Sachleistungen und vergleichbare Dienstleistungen gewähren, die Ausgaben zu tragen. Einen - auf einen Vorschlag des Sachverständigen Hans Meyer zurückgehenden - Antrag von Bündnis 90/Die Grünen im Rechtsausschuss, den Art. 104a Abs. 3 und 4 eindeutiger zu fassen, lehnten die Koalitionsfraktionen sogar ab (BT-Drs. 16/2069: 11, 40).

Wenn das Bundesverfassungsgericht also die vergleichbaren Dienstleistungen weit auslegt, die Zustimmungserfordernis auch bei geringer Kostenbelastung der Länder bejaht und/oder dem Bund das Recht abspricht, sich im Falle von geldwerten Sachleistungen und vergleichbaren Dienstleistungen von der Zustimmungserfordernis freizukaufen, hätte die Föderalismusreform ihr Kernziel, nämlich die Anzahl zustimmungsbedürftiger Gesetze zu reduzieren, mit hoher Wahrscheinlichkeit verfehlt - die Politikverflechtungsfalle hätte doch zugeschnappt.

Doch selbst wenn die Reform die Notwendigkeit einer Zustimmung des Bundesrates tatsächlich auf Dauer substanziell senkt, wird es vermutlich kaum zum viel zitierten Durchregieren der Bundesregierung kommen. Vielmehr wird vermutlich auch weiterhin der Dauerwahlkampf, dem sich die Regierungen in der Bundesrepublik ausgesetzt sehen, kohärente und weitreichende Reformen erschweren. 


\section{Literatur}

Bandelow, Nils/Hartmann, Anja, 2007: Weder Rot noch Grün. Machterosion und Interessenfragmentierung bei Staat und Verbänden in der Gesundheitspolitik, in: Egle/Zohlnhöfer 2007, 334-354.

Benz, Arthur, 2005: Kein Ausweg aus der Politikverflechtung? - Warum die Bundesstaatskommission scheiterte, aber nicht scheitern musste, in: PVS 46, 204-214.

Beyme, Klaus von, 1998: Interest Groups in Parliamentary Decisions in the German Bundestag, in: Government and Opposition 33 (1), 38-55.

Blancke, Susanne/Schmid, Josef, 2003: Bilanz der Bundesregierung Schröder in der Arbeitsmarktpolitik 1998-2002: Ansätze zu einer doppelten Wende, in: Egle/Ostheim/Zohlnhöfer 2003, 215-238.

Burkhart, Simone, 2008: Blockierte Politik. Ursachen und Folgen von „Divided Government" in Deutschland, Frankfurt a. M./New York.

Burkhart, Simone/Manow, Philip, 2006: Was bringt die Föderalismusreform? Wahrscheinliche Effekte der geänderten Zustimmungspflicht, Köln (Max-Planck-Institut für Gesellschaftsforschung, Working Paper 06/6).

Busch, Andreas, 2003: Extensive Politik in den Klippen der Semisouveränität: Die Innen- und Rechtspolitik der rot-grünen Koalition, in: Egle/ Ostheim/Zohlnhöfer 2003, 305-327.

Busch, Andreas, 2006: Verfassungspolitik: Stabilität und permanentes Austarieren, in: Manfred G. Schmidt/Reimut Zohlnhöfer (Hrsg.), Regieren in der Bundesrepublik Deutschland. Innen- und Außenpolitik seit 1949, Wiesbaden, 33-56.

Egle, Christoph/Ostheim, Tobias/Zohlnhöfer, Reimut (Hrsg.), 2003: Das rotgrüne Projekt: Eine Bilanz der Regierung Schröder 1998-2002, Wiesbaden.

Egle, Christoph/Zohlnhöfer, Reimut (Hrsg.), 2007: Ende des rot-grünen Projektes: Eine Bilanz der Regierung Schröder 2002-2005, Wiesbaden.

Feldkamp, Michael F. unter Mitarbeit von Birgit Ströbel, 2005: Datenhandbuch zur Geschichte des Deutschen Bundestages 1994 bis 2003, Baden-Baden.

Georgii, Harald/Borhanian, Sarab, 2006: Zustimmungsgesetze nach der Föderalismusreform: Wie hätte sich der Anteil der Zustimmungsgesetze verändert, wenn die vorgeschlagene Reform bereits 1998 in Kraft gewesen wäre?, Berlin.

Hildebrandt, Achim/Wolf, Frieder (Hrsg.), 2008: Die Politik der Bundesländer. Staatstätigkeit im Vergleich, Wiesbaden. 
Höreth, Marcus, 2007: Zur Zustimmungsbedürftigkeit von Bundesgesetzen: Eine kritische Bilanz nach einem Jahr Föderalismusreform I, in: ZParl $38,712-733$.

Höreth, Marcus, 2008: Gescheitert oder doch erfolgreich? Über die kontroverse Beurteilung der ersten Stufe der Föderalismusreform, in: Jahrbuch des Föderalismus 9, 139-159.

Ismayr, Wolfgang, 2008: Gesetzgebung im politischen System Deutschlands, in: ders. (Hrsg.), Gesetzgebung in Westeuropa: EU-Staaten und Europäische Union, Wiesbaden, 383-429.

Kinzig, Jörg/Steinhilber, Benjamin, 2008: Der Strafvollzug in der Hand der Bundesländer: Entwicklung und erste Auswirkungen einer (mutmaßlich missglückten) Reform, in: Jahrbuch des Föderalismus 9, 188-203.

Kluth, Winfried, 2007: Art. 74 GG, in: ders. (Hrsg.), Föderalismusreformgesetz. Einführung und Kommentierung, Baden-Baden, 162-178.

Kneip, Sascha, 2007: Anschieber oder Bremser? Das Bundesverfassungsgericht und die Reformpolitik der rot-grünen Bundesregierung, in: Egle/ Zohlnhöfer 2007, 215-238.

Lanzendorf, Ute/Pasternack, Peer, 2008: Landeshochschulpolitiken, in: Hildebrandt/Wolf 2008, 43-66.

Leunig, Sven, 2008: Landespolitik nach der Föderalismusreform I. Vortrag gehalten bei der Tagung „Föderalismusreform in Deutschland - Zwischenbilanz und Ausblick“ der Sektion „Regierungssystem und Regieren in der Bundesrepublik Deutschland“ der DVPW, Lüneburg, 25./26.09.08.

Meyer, Hans, 2006: Einige Überlegungen zum Entwurf einer Verfassungsänderung, Berlin (Stellungnahme für die Anhörung des Rechtsausschusses, 08.05.06).

Münch, Ursula, 2008: Materielles Abweichungsrecht der Länder und föderative Asymmetrien in der bundesdeutschen Bildungspolitik, in: Jahrbuch des Föderalismus 8 (2007), 224-237.

Rahm, Christian, 2008: Die Umsetzung der Föderalismusreform I am Beispiel des öffentlichen Wohnungsrechts in Bayern, in: Jahrbuch des Föderalismus 9, 204-215.

Raschke, Joachim, 2001: Die Zukunft der Grünen: „So kann man nicht regieren", Frankfurt a. M./New York.

Reutter, Werner, 2006: Föderalismusreform und Gesetzgebung, in: ZPol 16, 1249-1274.

Reutter, Werner, 2007: Struktur und Dauer der Gesetzgebungsverfahren des Bundes, in: ZParl 38, 299-315. 
Risse, Horst, 2007: Zur Entwicklung der Zustimmungsbedürftigkeit von Bundesgesetzen nach der Föderalismusreform 2006, in: ZParl 38, 707-712.

Rothgang, Heinz/Wessel, Anna Caroline, 2008: Sozialpolitik in den Bundesländern, in: Hildebrandt/Wolf 2008, 137-172.

Rürup, Matthias, 2008: Schulpolitik im deutschen Bundesstaat - zwei Jahre nach der Grundgesetznovelle 2006, in: Jahrbuch des Föderalismus 9, 172-187.

Sandberger, Georg, 2008: Die Umsetzung der Föderalismusreform im Hochschulbereich, in: Jahrbuch des Föderalismus 9, 160-171.

Scharpf, Fritz W., 1985: Die Politikverflechtungs-Falle: Europäische Integration und deutscher Föderalismus im Vergleich, in: PVS 26, 323-356.

Scharpf, Fritz W., 2006: Thesenpapier zur Anhörung des Rechtsausschusses zum Thema „Föderalismusreform“ am 15./16. Mai 2006, Berlin (Stellungnahme für die Anhörung des Rechtsausschusses, 12.05.06).

Scharpf, Fritz W., 2007a: Mit dieser Reform ist kein Staat zu machen, in: Max-Planck-Forschung, Heft 1, 15-18.

Scharpf, Fritz W., 2007b: Nicht genutzte Chancen der Föderalismusreform, in: Egle/Zohlnhöfer 2007, 197-214.

Schindler, Peter, 1999: Datenhandbuch zur Geschichte des Deutschen Bundestages 1949 bis 1999, Baden-Baden.

Schmidt, Manfred G., 2005: Gesamtbetrachtung, in: ders. (Hrsg.), Geschichte der Sozialpolitik in Deutschland seit 1945. Band 7: Bundesrepublik Deutschland 1982-1989. Finanzielle Konsolidierung und institutionelle Reform, Baden-Baden, 749-811.

Wolf, Frieder, 2008: Die Schulpolitik - Kernbestand der Kulturhoheit, in: Hildebrandt/Wolf 2008, 21-41.

Wolf, Frieder/Henkes, Christian, 2007: Die Bildungspolitik von 2002 bis 2005: Eine Misserfolgsgeschichte und ihre Ursachen, in: Egle/Zohlnhöfer 2007, 355-378.

Zohlnhöfer, Reimut, 2001: Die Wirtschaftspolitik der Ära Kohl. Eine Analyse der Schlüsselentscheidungen in den Politikfeldern Finanzen, Arbeit und Entstaatlichung, 1982-1998, Opladen.

Zohlnhöfer, Reimut, 2003: Rot-grüne Regierungspolitik in Deutschland 1998-2002. Versuch einer Zwischenbilanz, in: Egle/Ostheim/Zohlnhöfer 2003, 399-419.

Zohlnhöfer, Reimut, 2004: Die Wirtschaftspolitik der rot-grünen Koalition: Ende des Reformstaus?, in: ZPol 14, 381-402. 
Zohlnhöfer, Reimut, 2008: Föderalismusreform und die Entwicklung der Zustimmungsbedürftigkeit von Bundesgesetzen. Versuch einer Klärung, in: ZParl 39, 415-419.

Zohlnhöfer, Reimut/Egle, Christoph, 2007: Der Episode zweiter Teil - ein Überblick über die 15. Legislaturperiode, in: Egle/Zohlnhöfer 2007, 11-25.

Korrespondenzanschrift:

Prof. Dr. Reimut Zohlnhöfer

Professur für Politikwissenschaft, insbesondere international vergleichende Politikfeldanalyse

Universität Bamberg

Feldkirchenstr. 21

96052 Bamberg

E-Mail: reimut.zohlnhoefer@uni-bamberg.de

Web: http://www.uni-bamberg.de/policy

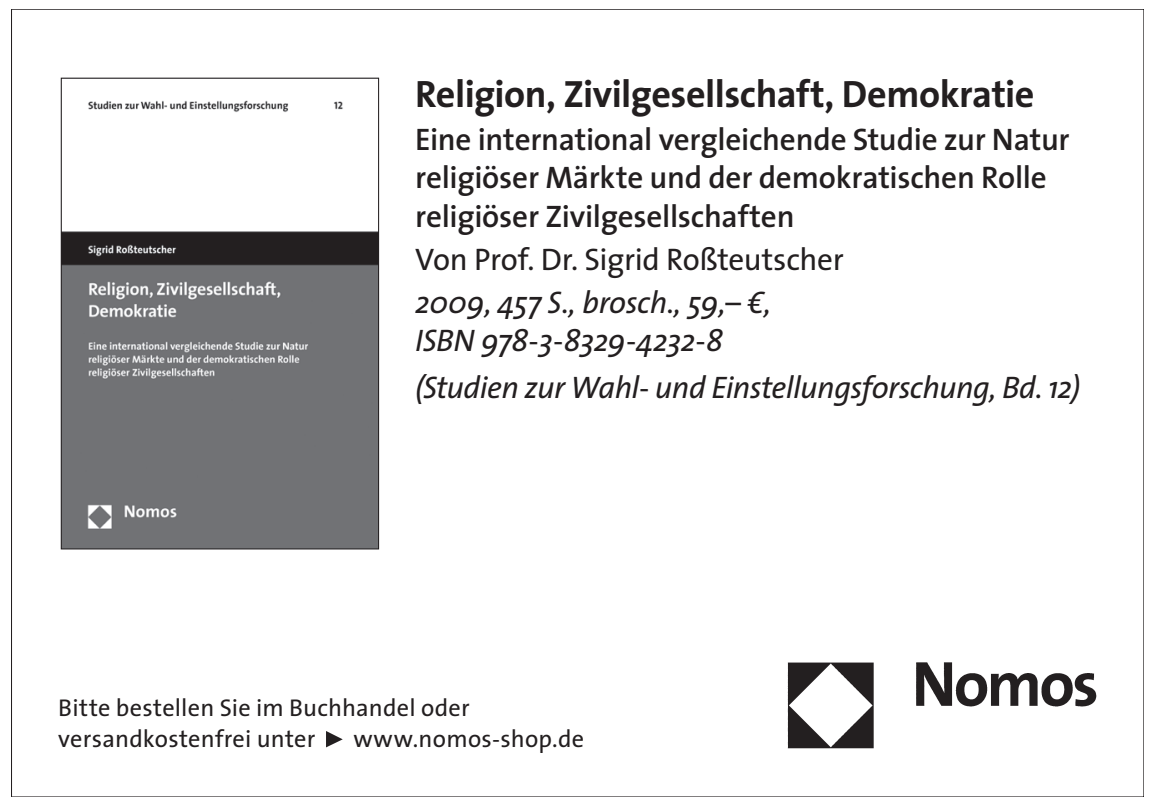




\section{Anhang}

Schlüsselentscheidungen 1998-2005 und Zustimmungsbedürftigkeit vor und nach der Föderalismusreform I

\begin{tabular}{|c|c|c|c|}
\hline \multirow[t]{2}{*}{ Nr. } & \multirow[t]{2}{*}{ Reform } & \multicolumn{2}{|c|}{ Zustimmungsbedürftig } \\
\hline & & $\begin{array}{l}\text { vor der } \\
\text { Reform }\end{array}$ & $\begin{array}{l}\text { nach der } \\
\text { Reform }\end{array}$ \\
\hline & Arbeitsmarktpolitik & & \\
\hline 1 & $\begin{array}{l}\text { Gesetz zur Neuregelung der geringfügigen } \\
\text { Beschäftigungsverhältnisse }\end{array}$ & $\mathrm{Ja}$ & $\mathrm{Ja}$ \\
\hline 2 & Teilzeit- und Befristungsgesetz & Nein & Nein \\
\hline 3 & Job-Aqtiv-Gesetz & Nein & Nein \\
\hline 4 & $\begin{array}{l}\text { Gesetz zur Reform des Betriebsverfassungs- } \\
\text { gesetzes }\end{array}$ & Nein & Nein \\
\hline 5 & $\begin{array}{l}\text { Erstes Gesetz für moderne Dienstleistungen } \\
\text { am Arbeitsmarkt (Hartz I) }\end{array}$ & $\begin{array}{l}\text { Nein (Ja } \\
\text { laut BRat) }\end{array}$ & Nein \\
\hline 6 & $\begin{array}{l}\text { Zweites Gesetz für moderne Dienstleistungen } \\
\text { am Arbeitsmarkt (Hartz II) }\end{array}$ & $\mathrm{Ja}$ & $\mathrm{Ja}$ \\
\hline 7 & $\begin{array}{l}\text { Drittes Gesetz für moderne Dienstleistungen } \\
\text { am Arbeitsmarkt (Hartz III) }\end{array}$ & Nein & Nein \\
\hline 8 & $\begin{array}{l}\text { Viertes Gesetz für moderne Dienstleistungen } \\
\text { am Arbeitsmarkt (Hartz IV) }\end{array}$ & $\mathrm{Ja}$ & $\mathrm{Ja}$ \\
\hline 9 & Gesetz zu Reformen am Arbeitsmarkt & Nein & Nein \\
\hline 10 & Berufsausbildungssicherungsgesetz & $\begin{array}{l}\text { Nein (Ja } \\
\text { laut BRat) }\end{array}$ & $\begin{array}{l}\text { Nein (Ja } \\
\text { laut BRat) }\end{array}$ \\
\hline & Auswärtiges, Europa und Sicherheit & & \\
\hline 11 & Gesetz zum Vertrag von Nizza & $\mathrm{Ja}$ & $\mathrm{Ja}$ \\
\hline 12 & Bundeswehrneuausrichtungsgesetz & Nein & Nein \\
\hline 13 & EU-Beitrittsvertragsgesetz & $\begin{array}{l}\text { Nein (Ja } \\
\text { lt. BRat) }\end{array}$ & $\begin{array}{l}\text { Nein }(\mathrm{Ja} \\
\text { lt. BRat) } \\
\end{array}$ \\
\hline 14 & Parlamentsbeteiligungsgesetz & Nein & Nein \\
\hline 15 & $\begin{array}{l}\text { Gesetz zu dem Vertrag vom 29. Oktober } 2004 \\
\text { über eine Verfassung für Europa }\end{array}$ & $\mathrm{Ja}$ & $\mathrm{Ja}$ \\
\hline & Bau/Verkehr & & \\
\hline 16 & Mietrechtsreformgesetz & Nein & Nein \\
\hline 17 & Gesetz zur Reform des Wohnungsbaurechts & $\mathrm{Ja}$ & Nein \\
\hline
\end{tabular}


Effekte der Föderalismusreform I

\begin{tabular}{|c|c|c|c|}
\hline \multirow[t]{2}{*}{$\mathrm{Nr}$. } & \multirow[t]{2}{*}{ Reform } & \multicolumn{2}{|c|}{ Zustimmungsbedürftig } \\
\hline & & $\begin{array}{l}\text { vor der } \\
\text { Reform }\end{array}$ & $\begin{array}{l}\text { nach der } \\
\text { Reform }\end{array}$ \\
\hline 18 & $\begin{array}{l}\text { Gesetz zur Einführung von streckenbezoge- } \\
\text { nen Gebühren für die Benutzung von Bundes- } \\
\text { autobahnen mit schweren Nutzfahrzeugen }\end{array}$ & $\mathrm{Ja}$ & Nein \\
\hline & Bildungspolitik & & \\
\hline 19 & $\begin{array}{l}\text { Fünftes Gesetz zur Änderung des Hochschul- } \\
\text { rahmengesetzes }\end{array}$ & $\begin{array}{l}\text { Ja (BVer- } \\
\text { fG) }\end{array}$ & $\begin{array}{l}\text { Kompe- } \\
\text { tenz } \\
\text { entfallen }\end{array}$ \\
\hline 20 & Professorenbesoldungsreformgesetz & $\mathrm{Ja}$ & $\begin{array}{l}\text { Kompe- } \\
\text { tenz } \\
\text { entfallen }\end{array}$ \\
\hline 21 & $\begin{array}{l}\text { Sechstes Gesetz zur Änderung des Hochschul- } \\
\text { rahmengesetzes }\end{array}$ & $\begin{array}{l}\text { Nein (Ja } \\
\text { lt. BRat) }\end{array}$ & $\begin{array}{l}\text { Kompe- } \\
\text { tenz } \\
\text { entfallen }\end{array}$ \\
\hline 22 & $\begin{array}{l}\text { Zwanzigstes Gesetz zur Änderung des Bun- } \\
\text { desausbildungsförderungsgesetzes } \\
\text { (20. BAföGÄndG) }\end{array}$ & $\mathrm{Ja}$ & $\mathrm{Ja}$ \\
\hline 23 & $\begin{array}{l}\text { Gesetz zur Änderung dienst- und arbeitsrecht- } \\
\text { licher Vorschriften im Hochschulbereich }\end{array}$ & Nein & $\begin{array}{l}\text { Kompetenz } \\
\text { weitge- } \\
\text { hend } \\
\text { entfallen }\end{array}$ \\
\hline 24 & Tagesbetreuungsausbaugesetz & $\begin{array}{l}\text { Nein (Ja } \\
\text { laut BRat) }\end{array}$ & $\begin{array}{l}\text { Ja (Art. } \\
104 a I V)\end{array}$ \\
\hline & Finanzpolitik & & \\
\hline 25 & Steuerreform 1999 & $\mathrm{Ja}$ & $\mathrm{Ja}$ \\
\hline 26 & Steuerreform 2000 & $\mathrm{Ja}$ & $\mathrm{Ja}$ \\
\hline 27 & Haushaltssanierungsgesetz 2000 & Nein & Nein \\
\hline 28 & $\begin{array}{l}\text { Gesetz zur Änderung des Wohngeldgesetzes } \\
\text { und anderer Gesetze (inhaltlich zum Haus- } \\
\text { haltssanierungsgesetz gehörend) }\end{array}$ & Ja & $\mathrm{Ja}$ \\
\hline 29 & Maßstäbegesetz & $\mathrm{Ja}$ & $\mathrm{Ja}$ \\
\hline 30 & Solidarpakt II & $\mathrm{Ja}$ & $\mathrm{Ja}$ \\
\hline 31 & Steuervergünstigungsabbaugesetz & $\mathrm{Ja}$ & $\mathrm{Ja}$ \\
\hline 32 & Gewerbesteuerreform & $\mathrm{Ja}$ & $\mathrm{Ja}$ \\
\hline 33 & Alterseinkünftegesetz & $\mathrm{Ja}$ & $\mathrm{Ja}$ \\
\hline
\end{tabular}




\begin{tabular}{|c|c|c|c|}
\hline \multirow[t]{2}{*}{ Nr. } & \multirow[t]{2}{*}{ Reform } & \multicolumn{2}{|c|}{ Zustimmungsbedürftig } \\
\hline & & $\begin{array}{l}\text { vor der } \\
\text { Reform }\end{array}$ & $\begin{array}{l}\text { nach der } \\
\text { Reform }\end{array}$ \\
\hline & Innen- und Rechtspolitik & & \\
\hline 34 & $\begin{array}{l}\text { Gesetz zur Reform des Staatsangehörigkeits- } \\
\text { rechts }\end{array}$ & $\mathrm{Ja}$ & Nein \\
\hline 35 & Lebenspartnerschaftsgesetz & Nein & Nein \\
\hline 36 & Lebenspartnerschaftsgesetzergänzungsgesetz & $\mathrm{Ja}$ & $\begin{array}{l}\text { Ja (Kom- } \\
\text { petenz } \\
\text { zum Teil } \\
\text { entfallen) }\end{array}$ \\
\hline 37 & Zuwanderungsgesetz (14.WP) & $\mathrm{Ja}(84 \mathrm{I})$ & $\begin{array}{l}\mathrm{Ja}(104 \mathrm{a} \\
\mathrm{IV})\end{array}$ \\
\hline 38 & $\begin{array}{l}\text { Gesetz zur Durchsetzung der Gleichstellung } \\
\text { von Frauen und Männern }\end{array}$ & Nein & Nein \\
\hline 39 & $\begin{array}{l}\text { Gesetz zur Bekämpfung des internationalen } \\
\text { Terrorismus }\end{array}$ & $\mathrm{Ja}$ & Nein \\
\hline 40 & Stammzellgesetz & Nein & Nein \\
\hline 41 & Erstes Gesetz zur Modernisierung der Justiz & Nein & Nein \\
\hline 42 & $\begin{array}{l}\text { Gesetz zur Regelung des Zugangs zu } \\
\text { Informationen des Bundes } \\
\text { (Informationsfreiheitsgesetz - IFG) }\end{array}$ & Nein & Nein \\
\hline 43 & Luftsicherheitsgesetz & $\begin{array}{l}\text { Nein (Ja } \\
\text { laut BRat) }\end{array}$ & $\begin{array}{l}\text { Nein (Ja } \\
\text { laut BRat) }\end{array}$ \\
\hline 44 & Zuwanderungsgesetz (15. WP) & $\begin{array}{l}\mathrm{Ja} \\
(84 \mathrm{I}) \\
\end{array}$ & $\begin{array}{l}\text { Ja } \\
(104 a \mathrm{IV})\end{array}$ \\
\hline 45 & Europäisches Haftbefehlsgesetz & $\begin{array}{l}\text { Nein (Ja } \\
\text { laut BRat) }\end{array}$ & $\begin{array}{l}J a \\
(104 a I V)\end{array}$ \\
\hline 46 & Prostitutionsgesetz & Nein & Nein \\
\hline 47 & Antidiskriminierungsgesetz (gescheitert) & Nein & Nein \\
\hline & Landwirtschaft und Verbraucherschutz & & \\
\hline 48 & BSE-Maßnahmengesetz & $\mathrm{Ja}$ & Nein \\
\hline 49 & Verbraucherinformationsgesetz (gescheitert) & $\mathrm{Ja}$ & Nein \\
\hline
\end{tabular}


Effekte der Föderalismusreform I

\begin{tabular}{|c|c|c|c|}
\hline \multirow[t]{2}{*}{ Nr. } & \multirow[t]{2}{*}{ Reform } & \multicolumn{2}{|c|}{ Zustimmungsbedürftig } \\
\hline & & $\begin{array}{l}\text { vor der } \\
\text { Reform }\end{array}$ & $\begin{array}{l}\text { nach der } \\
\text { Reform }\end{array}$ \\
\hline & Sozialpolitik & & \\
\hline 50 & $\begin{array}{l}\text { Gesetz zu Korrekturen in der Sozialversiche- } \\
\text { rung und zur Sicherung der Arbeitnehmer- } \\
\text { rechte }\end{array}$ & $\mathrm{Ja}$ & $\mathrm{Ja}$ \\
\hline 51 & GKV-Solidaritätsstärkungsgesetz & $\mathrm{Ja}$ & Nein \\
\hline 52 & $\begin{array}{l}\text { Drittes Gesetz zur Änderung des Bundeser- } \\
\text { ziehungsgeldgesetzes }\end{array}$ & $\mathrm{Ja}$ & $\mathrm{Ja}$ \\
\hline 53 & Altersvermögensgesetz (Riester-Rente) & $\mathrm{Ja}$ & $\mathrm{Ja}$ \\
\hline 54 & Altersvermögensergänzungsgesetz & Nein & Nein \\
\hline 55 & Gesundheitsreformgesetz 2000 & $\mathrm{Ja}$ & Nein \\
\hline 56 & Beitragssatzsicherungsgesetz & $\begin{array}{l}\text { Nein (Ja } \\
\text { lt. BRat) }\end{array}$ & Nein \\
\hline 57 & $\begin{array}{l}\text { Zwölftes SGB V-Änderungsgesetz (inhaltlich } \\
\text { zum Beitragssatzsicherungsgesetz gehörend) }\end{array}$ & Ja & Nein \\
\hline 58 & Rentenversicherungsnachhaltigkeitsgesetz & Nein & Nein \\
\hline 59 & GKV-Modernisierungsgesetz & $\mathrm{Ja}$ & Nein \\
\hline 60 & $\begin{array}{l}\text { Gesetz zur Einordnung des Sozialhilferechts } \\
\text { in das Sozialgesetzbuch }\end{array}$ & $\mathrm{Ja}$ & $\mathrm{Ja}$ \\
\hline 61 & $\begin{array}{l}\text { Zweites Gesetz zur Änderung des Sechsten } \\
\text { Buches Sozialgesetzbuch und anderer Gesetze }\end{array}$ & Nein & Nein \\
\hline 62 & $\begin{array}{l}\text { Gesetz zur Berücksichtigung der Kinder- } \\
\text { erziehung im Beitragsrecht der sozialen Pfle- } \\
\text { geversicherung (Kinder-Berücksichtigungs- } \\
\text { gesetz) }\end{array}$ & Nein & Nein \\
\hline & Umweltpolitik & & \\
\hline 63 & Ökologische Steuerreform & Nein & Nein \\
\hline 64 & Erneuerbare-Energien-Gesetz & $\mathrm{Ja}$ & Nein \\
\hline 65 & Kraft-Wärme-Kopplungsgesetz & Nein & Nein \\
\hline 66 & $\begin{array}{l}\text { Gesetz zur Neuregelung des Rechts des Na- } \\
\text { turschutzes und der Landschaftspflege }\end{array}$ & Nein & Nein \\
\hline 67 & $\begin{array}{l}\text { Gesetz zur geordneten Beendigung der Kern- } \\
\text { energienutzung zur gewerblichen Erzeugung } \\
\text { von Elektrizität }\end{array}$ & Nein & Nein \\
\hline 68 & Treibhausgas-Emissionshandelsgesetz & Ja & $\mathrm{Ja}$ \\
\hline
\end{tabular}




\begin{tabular}{|c|l|l|l|}
\hline Nr. & Reform & \multicolumn{2}{|l|}{ Zustimmungsbedürftig } \\
\hline & & $\begin{array}{l}\text { vor der } \\
\text { Reform }\end{array}$ & $\begin{array}{l}\text { nach der } \\
\text { Reform }\end{array}$ \\
\hline 69 & Hochwasserschutzgesetz & $\begin{array}{l}\text { Nein (Ja } \\
\text { laut BRat) }\end{array}$ & $\begin{array}{l}\text { Unklar } \\
\text { (vielleicht } \\
\text { Art. 104a } \\
\text { IV, denn } \\
\text { „den } \\
\text { Ländern } \\
\text { entstehen } \\
\text { nicht } \\
\text { quantifi- } \\
\text { zierbare } \\
\text { Kosten“) }\end{array}$ \\
& & & \\
& & & \\
& & & \\
\hline
\end{tabular}

Schlüsselentscheidungen im engeren Sinne sind kursiv formativ. Fett und kursiv sind Fälle markiert, bei denen sich durch die Reform die Zustimmungsbedürftigkeit verändert hat.

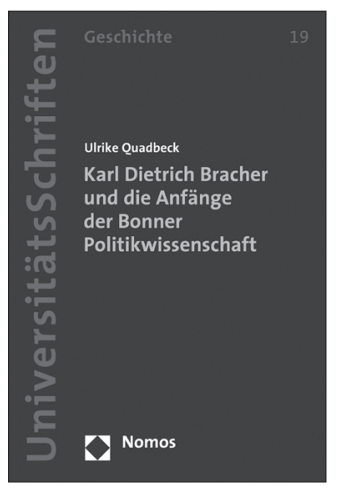

\section{Karl Dietrich Bracher und die Anfänge der Bonner Politikwissenschaft}

Von Dr. Ulrike Quadbeck

2008, 436 S., brosch., 69,- $€$, ISBN 978-3-8329-3740-9

(Nomos Universitätsschriften - Geschichte, Bd. 19)

Mit bahnbrechenden Untersuchungen sowie der richtungweisenden Unterscheidung zwischen Demokratie und Diktatur, hergeleitet aus den Erfahrungen des 20. Jahrhunderts, hat K. D. Bracher in den fünfziger/ sechziger Jahren seinen Ruf als Nestor der Zeitgeschichtsforschung gewonnen und dem Bonner Seminar für Politische Wissenschaft Orientierung gegeben. 